\title{
Targeting monocyte chemotactic protein-1 synthesis with bindarit induces tumor regression in prostate and breast cancer animal models
}

\author{
Massimo Zollo • Valeria Di Dato - Daniela Spano - Daniela De Martino • \\ Lucia Liguori • Natascia Marino • Viviana Vastolo • Luigi Navas • \\ Beatrice Garrone $\cdot$ Giorgina Mangano $\cdot$ Giuseppe Biondi $\cdot$ Angelo Guglielmotti
}

Received: 19 October 2011/Accepted: 22 March 2012/Published online: 7 April 2012

(C) Springer Science+Business Media B.V. 2012

\begin{abstract}
Prostate and breast cancer are major causes of death worldwide, mainly due to patient relapse upon disease recurrence through formation of metastases. Chemokines are small proteins with crucial roles in the immune system, and their regulation is finely tuned in early inflammatory responses. They are key molecules during inflammatory processes, and many studies are focusing on their regulatory functions in tumor growth and angiogenesis during metastatic cell seeding and spreading. Bindarit is an anti-inflammatory indazolic derivative that can inhibit the synthesis of MCP-1/CCL2, with a potential inhibitory function in tumor progression and metastasis formation. We show here that in vitro, bindarit can modulate cancercell proliferation and migration, mainly through negative regulation of TGF- $\beta$ and AKT signaling, and it can impair
\end{abstract}

Valeria Di Dato and Daniela Spano contributed equally to the work.

Electronic supplementary material The online version of this article (doi:10.1007/s10585-012-9473-5) contains supplementary material, which is available to authorized users.

M. Zollo $(\bowtie) \cdot$ V. Di Dato - D. Spano - D. De Martino .

L. Liguori · N. Marino · V. Vastolo · L. Navas

Centro di Ingegneria Genetica, Biotecnologie Avanzate

(CEINGE), Via Gaetano Salvatore 486, 80145 Naples, Italy

e-mail: massimo.zollo@unina.it

M. Zollo · V. Di Dato · D. Spano · D. De Martino ·

N. Marino $\cdot$ V. Vastolo

Dipartimento di Biochimica e Biotecnologie Mediche (DBBM),

"Federico II" University of Naples, Naples, Italy

L. Navas

Dipartimento di Scienze Cliniche Veterinarie, Sez. di Clinica

Chirurgica, "Federico II" University of Naples, Naples, Italy

B. Garrone · G. Mangano - G. Biondi · A. Guglielmotti

Angelini Research Centre, S. Palomba-Pomezia, Rome, Italy the NF- $\kappa \mathrm{B}$ signaling pathway through enhancing the expression of the NF- $\kappa$ B inhibitor IkB- $\alpha$. In vivo administration of bindarit results in impaired metastatic disease in prostate cancer xenograft mice (PC-3M-Luc2 cells injected intra-cardially) and impairment of local tumorigenesis in syngeneic Balb/c mice injected under the mammary gland with murine breast cancer cells (4T1-Luc cells). In addition, bindarit treatment significantly decreases the infiltration of tumor-associated macrophages and myeloid-derived suppressor cells in 4T1-Luc primary tumors. Overall, our data indicate that bindarit is a good candidate for new therapies against prostate and breast tumorigenesis, with an action through impairment of inflammatory cell responses during formation of the tumor-stroma niche microenvironment.

Keywords Prostate $\cdot$ Breast $\cdot$ Tumor-associated macrophages - Myeloid-derived suppressor cells . MCP-1/CCL2 Xenograft

\section{Introduction}

Metastasis is a multistep process in which malignant cells must initially escape from the primary tumor, and then invade the surrounding tissues, enter the vascular circulation, and successfully infiltrate into a secondary target site to proliferate and form a clinically relevant metastatic colony. Alternatively, metastatic cells can remain quiescent, potentially never proliferating beyond a microscopic focus of cells [1].

The ability of a tumor cell to successfully metastasize and establish a viable lesion at a secondary site is dependent on its interactions with and use of microenvironment milieu, to promote self-survival and proliferation. The cancer metastatic process initiates with transformation of 
primary tumor cells into an oncogenic phenotype that promotes unregulated growth, angiogenesis, breakdown of the extracellular milieu, intravasation, cell survival in the circulation, cell adhesion to the endothelium of a target organ, extravasation, and subsequent growth.

Several solid cancers have a predominant phenotype for the formation of bone metastases, including breast and prostate cancers. Over $70 \%$ of such patients will develop bone metastases, for which there is at present no cure. They then show disease progression with a bad quality of life expected because of the associated complications, such as bone absorption, intractable pain, spinal cord compression, long bone fractures, and refractory hypercalcemia. Most importantly, patients affected by bone metastasis show significantly longer mean survival times than those with metastases to other organs, and thus can suffer longer periods of metastasis-induced pain.

In the promotion of tumor growth, cancer cells themselves provide the growth factors, cytokines and chemokines that enhance cell proliferation and invasiveness, and induce angiogenesis, inflammation and the recruitment of immune cells, including tumor-associated macrophages (TAMs) and myeloid-derived suppressor cells (MDSCs). These immune cells can represent up to $50 \%$ of a tumor mass, and they are known to have fundamental roles in disease progression [2].

The NF-kB pathway mediates pro-inflammatory signaling and promotes the proliferation and migration of tumor cells [3]. Many pro-inflammatory genes are transcriptionally regulated by nuclear factor-kappa B (NF-kB), such as monocyte chemoattractant protein-1 (MCP-1, also known as chemokine ligand 2 [CCL2], and referred to as MCP-1 henceforth), which is a chemotactic factor for monocytes and $\mathrm{T}$ lymphocytes.

Recently, MCP-1 and its receptor CCR2 have been shown to have key roles in the promotion of tumorigenesis and metastasis formation $[4,5]$. In breast cancer, MCP-1 expression has been associated with advanced disease state, tumor progression, and angiogenesis, and in particular to lung metastasis formation [6-9]. MCP-1 and CCR2 are involved in the regulation of cell migration, cell proliferation and tumor growth in breast cancer, multiple myeloma and prostate carcinoma [7, 10], where they influence the development of bone-targeted metastases. Indeed, several of the cell types found in the bone marrow compartment have been shown to synthesize and secrete MCP-1, where it contributes to the development of the metastatic niche via indirect effects on metastasis through stimulation of angiogenesis and monocyte/macrophage recruitment [8, $11,12]$. The blocking of MCP-1 activity can also result in significant prolongation of survival of immune-deficient mice that have human breast or prostate carcinomas $[4,13$, 14]. Furthermore, it has recently been shown that MCP-1 can modulate Th2 tumor-reactive T-cell polarization and can inhibit the generation of these Th2 cells. For all of these reasons, MCP-1 appears to be a novel target for the treatment of patients with metastatic bone disease [15].

Bindarit (2-methyl-2-[[1-(phenylmethyl)-1H-indazol-3yl] methoxy] propanoic acid) is an indazolic derivative that inhibits the production of the MCP subfamily of the inflammatory chemokines MCP-1 (CCL2), MCP-2 (CCL8) and MCP-3 (CCL7) [16]. The effects of bindarit are mediated by the down-regulation of the classical NF-kB signaling pathway, which involves reduction in $\mathrm{IkB} \alpha$ and $\mathrm{p} 65$ phosphorylation and reduced activation of NF-kB dimers, and the subsequently reduced nuclear translocation and DNA-binding of p65 [17]. In vivo, the therapeutic effects of bindarit in a number of experimental models of inflammatory diseases, including nephritis, arthritis, pancreatitis, colitis and vascular damage, have been associated with its ability to inhibit MCP-1 production and selectively interfere with monocyte/macrophage recruitment and the early inflammatory response [18-25]. Furthermore, studies in human-melanoma-bearing mice, in which the MCP-1overexpressing melanoma cells are subcutaneously injected into nude mice, have indicated that bindarit can reduce tumor growth and macrophage recruitment through inhibition of MCP-1 [26].

Here, we further examined the clinic potential of bindarit in the prevention of tumorigenesis and metastasis in prostate and breast cancer xenograft animal models. Our findings provide evidence that bindarit impairs not only local tumorigenesis, as previously demonstrated in melanoma xenograft models, but also metastasis formation. Moreover, our data provide evidence that bindarit impairs the tumor microenvironment by preventing infiltration not only of TAMs, as previously described [26], but also of MDSCs. Finally, in vivo, bindarit impairs the organization of endothelial cells in the formation of blood vessels, thus impairing the tumor angiogenesis. These mechanisms of action determine an increased survival of cancer xenograft animal models. These data suggest that bindarit is a promising agent for the treatment of prostate cancer and breast cancer.

\section{Materials and methods}

\section{Drug preparation}

Bindarit (molecular weight, 324.38) was synthesized by Angelini (Aziende Chimiche Riunite Angelini Francesco [ACRAF], Italy). For in vitro experiments, bindarit was prepared as a $100 \mathrm{mM}$ stock solution in $1 \mathrm{~N} \mathrm{NaOH}$, which was diluted to the tested concentrations in phosphate-buffered saline (PBS) at $\mathrm{pH}$ 7.4. For in vivo experiments, 
bindarit was prepared as a suspension in $0.5 \%$ methylcellulose (MTC) aqueous solution.

Cell culture

The PC-3M-Luc2 human prostate cancer cell line is a luciferase (Luc)-expressing cell line that was grown in minimum essential Eagle's medium (MEM-Eagle; Sigma) supplemented with $2 \mathrm{mM}$ L-glutamine, $10 \%$ fetal bovine serum, $100 \mathrm{U} / \mathrm{ml}$ penicillin and $100 \mu \mathrm{g} / \mathrm{ml}$ streptomycin, at $37{ }^{\circ} \mathrm{C}$ in a $95 \%$ air and $5 \% \mathrm{CO}_{2}$ humidified incubator. The cells were purchased from Caliper Life Sciences under a contract for their use for experimental research only in the Dipartimento di Biochimica e Biotecnologie Mediche, at the "Federico II" University of Naples, and in agreement with the Centro di Ingegneria Genetica Biotecnologie Avanzate (CEINGE) of Naples. The mouse 4T1-Luc breast cancer cell line was obtained in-house by transfecting the parent cells with a pCMV-GL-4 plasmid expressing the Luc reporter gene under the control of a strong CMV promoter, and by selecting the Luc-expression-stable clones using a methodology previously described [27]. These Luc-expressing cell lines retain similar properties to their parental cell lines (with respect to cell growth, cell cycle and cell invasion), and they were maintained according to the parental line protocols: Dulbecco's modified eagle's medium (DMEM; Gibco-Invitrogen) supplemented with $2 \mathrm{mM}$ L-glutamine, $10 \%$ fetal bovine serum, $100 \mathrm{U} / \mathrm{ml}$ penicillin, and $100 \mu \mathrm{g} / \mathrm{ml}$ streptomycin, at $37{ }^{\circ} \mathrm{C}$ in a $95 \%$ air $5 \% \mathrm{CO}_{2}$ humidified incubator. The murine J774 macrophage cell line was also grown in DMEM (Gibco-Invitrogen) supplemented with $2 \mathrm{mM}$ L-glutamine, $10 \%$ fetal bovine serum, $100 \mathrm{U} / \mathrm{ml}$ penicillin, and $100 \mu \mathrm{g} / \mathrm{ml}$ streptomycin, at $37{ }^{\circ} \mathrm{C}$ in a $95 \%$ air $5 \% \mathrm{CO} 2$ humidified incubator.

Quantitative real-time polymerase chain reaction (qRT-PCR)

Total RNA was isolated from cells using Trizol reagent (Invitrogen) according to the manufacturer protocol. After measurement of RNA yield and quality using a NanoDrop machine (Celbio), the cDNA was synthesized by random hexamers with iScript cDNA synthesis kits (Bio-Rad), according to the protocols supplied by Bio-Rad. Two micrograms of total RNA in $20 \mu \mathrm{l}$ was used in each reaction. qRT-PCR was performed using the SYBR Green PCR Master Mix (Applied Biosystems) and the Applied Biosystems Model 7900HT sequence detection system, according to the protocols supplied by Applied Biosystems. The primers were designed with the Primer Express 2.1 program (Applied Biosystems). All of the primers were designed in two different exons, to avoid amplification of genomic DNA. All qRT-PCRs were performed in duplicate, with $70 \mathrm{ng}$ ss-cDNA used in each $10-\mu$ l reaction. $\beta$-Actin mRNA was used to normalize the mRNA concentrations. The primer sequences for the tested genes were the following:

h-MCP-1 forward, 5'-CATTCCCCAAGGGCTCG-3'; h-MCP-1 reverse, 5'-GCCTCTGCACTGAGATCTTCC TA-3';

h-MCP-2 forward, 5'-GTTTCTGCAGCGCTTCTGTG-3'; h-MCP-2 reverse, 5'-GCAGGTGATTGGAATGGAAACT-3';

h-MCP-3 forward, 5'-TGCTTTCAGCCCCCAGG-3'; h-MCP-3 reverse, 5'-CCAGCCTCTGCTTAGGGATT TT- $3^{\prime}$;

h- $\beta$-actin forward, $5^{\prime}$-GACCCAGATCATGTTTGAG ACCTT;

h- $\beta$-actin reverse, $5^{\prime}$-CCAGAGGCGTACAGGGATAG C- $3^{\prime}$;

m-MCP-1 forward, 5'-CAGCAGCAGGTGTCCCAAA-3'; m-MCP-1 reverse, 5'-TGTCTGGACCCATTCCTTCTT G-3';

m-MCP-2 forward, 5'-CCTGTCAGCCCAGAGAAGCT- $3^{\prime}$; m-MCP-2 reverse, 5'-TTTTCAGCACCCGAAGGG-3'; m-MCP-3 forward, 5'-CTTTCAGCATCCAAGTGTGG G-3';

m-MCP-3 reverse, 5'-GTAGCTCTTGAGATTCCTCT TGGG-3';

m- $\beta$-actin forward, 5'-AGGCCAACCGTGAAAAGA TG-3';

m- $\beta$-actin reverse, $5^{\prime}$-GCCTGGATGGCTACGTACAT G-3'.

Relative gene expression was calculated using the $2^{-\Delta \mathrm{Ct}}$ method, with $\Delta \mathrm{Ct}$ calculated using the differences in the mean $\mathrm{Ct}$ between the genes and their internal controls.

Cell proliferation assays

To assess the direct effects of bindarit on cell proliferation, proliferation assays were performed using Cell Titer $96^{\circledR}$ $\mathrm{AQ}_{\text {ueous }}$ One Solution cell proliferation assay kits, according to the manufacturer instructions. These kits use the novel tetrazolium compound 3-(4,5-dimethylthiazol2-yl)-5-(3-carboxymethoxyphenyl)-2-(4-sulfophenyl) (MTS reagent, Promega Corporation, Milan, Italy) to measure cell proliferation. PC-3M-Luc2 and 4T1-Luc cells were starved for $16 \mathrm{~h}$, and then seeded in quadruplicate in 96-well plates at densities of $3 \times 10^{3}$ and $2.5 \times 10^{3}$ per well, respectively. The cells were then treated with PBS (control) or with 50, 100, 200 and $300 \mu \mathrm{M}$ bindarit for 0,24 and $48 \mathrm{~h}$. The medium supplemented with PBS or bindarit was replaced twice daily. Wells with serum-free medium were used as the negative controls. For each experimental point, $20 \mu \mathrm{l}$ 
MTS reagent was added into each well, and the cells were incubated at $37^{\circ} \mathrm{C}$ for $2 \mathrm{~h}$. The absorbance values were then measured at $490 \mathrm{~nm}$ with a microplate reader (Victor ${ }_{\mathrm{TM}}^{3}$ Wallac 1420 Multilabel Counter, Perkin Elmer ${ }^{\circledR}$ ), and normalized with respect to $t=0 \mathrm{~h}$. For each experimental point, the means of normalized absorbance and their standard errors (SE) were calculated. A total of two independent sets of experiments were performed.

To determine the effects of bindarit on cell proliferation induced by immune cells, proliferation assays were performed on 4T1-Luc cells in the presence of conditioned medium from $\mathrm{J} 774$ cells stimulated with LPS and treated with bindarit or PBS. Briefly, $2 \times 10^{5} \mathrm{~J} 774$ cells were seeded in 6-well plates. After $24 \mathrm{~h}$, the cells were washed with PBS and treated for $16 \mathrm{~h}$ with $1 \mu \mathrm{g} / \mathrm{ml}$ LPS without or with $300 \mu \mathrm{M}$ bindarit, in DMEM containing $1 \%$ fetal bovine serum, $2 \mathrm{mM}$ L-glutamine, $100 \mathrm{U} / \mathrm{ml}$ penicillin and $100 \mu \mathrm{g} / \mathrm{ml}$ streptomycin, at $37{ }^{\circ} \mathrm{C}$ in a $95 \%$ air and $5 \%$ $\mathrm{CO}_{2}$ humidified incubator. The LPS treatment stimulates MCP-1 expression, while the concomitant treatment with $300 \mu \mathrm{M}$ bindarit down-regulates this expression [16, 17]. Then the conditioned media were used in cell proliferation assays. As described above, $2.5 \times 10^{3} 4 \mathrm{~T} 1$-Luc cells were seeded in quadruplicate in 96-well plates, allowed to attach to the plates, washed with PBS, and then grown in $100 \mu \mathrm{J} 774$ conditioned medium for 24 and $48 \mathrm{~h}$. Cell proliferation was assessed as described above. A total of two independent sets of experiments were performed.

\section{Flow cytometry}

The 4T1-Luc and PC-3M-Luc2 cells were treated with PBS (control) or bindarit $(50,100,200,300 \mu \mathrm{M})$ for $24 \mathrm{~h}$. The medium supplemented with PBS or bindarit was replaced twice daily. For the cell-cycle analysis, after treatment, the cells were resuspended in PBS containing $0.002 \%$ nonidet $\mathrm{P} 40,12.5 \mu \mathrm{g} / \mathrm{ml}$ ribonuclease $\mathrm{A}$, and $20 \mu \mathrm{g} / \mathrm{ml}$ propidium iodide, incubated at room temperature for $3 \mathrm{~h}$ in the dark, and analyzed on a FACScan flow cytometer (Becton-Dickinson Immunocytometry Systems). For each experimental point, the cell-cycle analysis was performed in duplicate, and two independent sets of experiments were performed.

For evaluation of the percentages of Annexin-V-positive cells, after $24 \mathrm{~h}$ treatment, $2 \times 10^{5} 4 \mathrm{~T} 1$-Luc and PC-3MLuc2 cells were incubated for $30 \mathrm{~min}$ with a FITC-conjugated anti-Annexin $\mathrm{V}$ antibody (BD Pharmingen) and analyzed using a BD FACSCanto II flow cytometer (BD Biosciences, Oxford, UK). The collected data were analyzed using the FACSDiva software, version 6 (BD Biosciences). Each experimental point was assessed in duplicate, and two independent sets of experiments were performed.
Migration assays

To assay the direct effects of bindarit on cell migration PC-3M-Luc2 and 4T1-Luc cells were resuspended to a density of $2.5 \times 10^{4}$ cells per $200 \mu \mathrm{l}$ in MEM-Eagle (PC-3M-Luc2 cells) or DMEM (4T1-Luc) in the presence of bindarit $(50,100,300 \mu \mathrm{M})$ or PBS (control). This cell suspension $(200 \mu \mathrm{l})$ was then added to the upper chamber of 8- $\mu \mathrm{m}$-pore-size Transwell inserts (Corning Incorporated, Costar, Corning, NY, USA). Each experimental point was assessed in duplicate. Five hundred microliters of medium containing $0.5 \%$ fetal bovine serum, which served as the chemoattractant, was added to the lower chamber of the wells, and the samples were incubated at $37{ }^{\circ} \mathrm{C}$ for $4 \mathrm{~h}$. Then the cells that had migrated to the lower membrane surface were fixed for $30 \mathrm{~min}$ with pre-cooled $\left(-20^{\circ} \mathrm{C}\right)$ $5 \%$ acetic acid in $95 \%$ ethanol, and stained with hematoxylin/eosin. To count the migrating cells, the membranes of each chamber were examined under optical microscopy, and the migrating cells were counted using the Cell Counter ImageJ program. For each experimental point, the mean $\pm \mathrm{SE}$ of the number of migrated cells per membrane were calculated. Two independent sets of experiments were carried out.

To assess the effects of bindarit on cell migration induced by immune cells, we performed motility assays on 4T1-Luc cells in the presence of conditioned medium from J774 cells stimulated with LPS and treated with PBS or bindarit, as above described. Five hundred microliters of J774 conditioned medium was put into the lower chamber and used as the chemoattractant in the motility assay. The 4T1-Luc cells $\left(2.5 \times 10^{4}\right)$ were seeded into the upper chamber in DMEM containing $1 \%$ fetal bovine serum, allowed to migrate for $4 \mathrm{~h}$ at $37^{\circ} \mathrm{C}$, and then fixed and counted as described above. Two independent sets of experiments were carried out.

\section{Caspase assay}

For the measurement of caspase-3 activity, the PC-3MLuc2 and 4T1-Luc cells were treated with PBS (control) or $10 \mu \mathrm{M}$ bindarit in their respective growth media for $8 \mathrm{~h}$ at $37^{\circ} \mathrm{C}$. Then $100 \mu \mathrm{g}$ protein extract from each treatment was used for the caspase- 3 activity measurements, using Ac-DEVD-AFC Caspase-3 Fluorigenic Substrate kits (BD Pharmagen), according to the manufacturer protocol.

Animal care

The mice were maintained on a purified standard diet (4RF21) from Mucedola (Settimo Milanese, Milan, Italy). They were housed at a maximum of four mice per cage, and maintained at a constant temperature $\left(22 \pm 1^{\circ} \mathrm{C}\right)$ and 
humidity $(45-75 \%)$ on a 12-h light/dark cycle, with food and water ad libitum. The mice were monitored daily by a vet, to be sure of their health and behavioral status.

All of the animal work was conducted according to the relevant national and international guidelines. Approval was obtained from the Institutional Animal Care and Ethical Committee at CEINGE and the "Federico II" University of Naples, according to Protocol \#29 of 30/09/2009, and the Italian Ministry of Health, Dipartimento Sanità Pubblica Veterinaria D.L. 116/92, which confirms that all of the experiments carried out conformed to the relevant regulatory standards.

In vivo xenografts and proof of concept treatment trials

A total of 30 male 7-week-old nude athymic mice (Harlan, Italy) were anesthetized with $3 \%$ Avertin (Sigma) and placed on a warm stage. These mice were then injected with $1.5 \times 10^{6}$ PC-3M-Luc2 cells per $100 \mu \mathrm{l}$ PBS into the left cardiac ventricle. Briefly, the cells were taken up into a 1 -ml syringe (without needle) and a 0.5 -inch 27 gauge needle was then attached to the syringe. A small air bubble was created on the plunger side of the syringe before cell injection. The needle was inserted into the second intercostal space, approximately $3 \mathrm{~mm}$ to the left of the sternum, and directed centrally. Cells were injected slowly, over 10-20 s. The cell-injection day was taken as the starting point and defined as day 0 . The mice were then injected with $15 \mathrm{mg} / \mathrm{ml} / \mathrm{kg}$ luciferin (Calipers Life Sciences), and 10 min after, the bioluminescent (BLI) signals of the injected cells were measured using an IVIS Imaging 3D Illumina System (Caliper Life Sciences).

Mice showing the appropriate positioning of injected cells after day 7 from injection were divided in two groups using similar BLI emission mean to overcome technical problems with injection of cells at day 0: group n.1 was treated without (MTC vehicle) and group n.2 was treated with bindarit $100 \mathrm{mg} / \mathrm{kg}$ ip $(5 \mathrm{ml} / \mathrm{kg}$ body weight). Treatments started on day 7 from the intracardiac injection, then each mice group were administered every day, once a day, for five consecutive days a week. BLI measurements were carried out once a week. At week 6 after the intracardiac injection, for those mice not showing any significantly robust BLI signal, the treatment was stopped, while the mice that were still showing significant BLI signals continued to receive treatment up to week 12. Quantification of the BLI signals was performed using the Living Image 3.2 program (Caliper Life Science).

At week 12, the number of live mice from each group was used to calculate the survival curve. Statistical analysis of the survival curve was performed using the StatView 5.0.1.0 program.
To study the inhibition of local tumorigenesis by bindarit, we established a syngeneic animal model of mouse breast carcinoma. Here, $2.5 \times 10^{5} 4 \mathrm{~T} 1$-Luc cells per $100 \mu \mathrm{l}$ PBS were orthotopically transplanted into the mammary fat pads of 10-week-old BALB/c mice (Harlan, Italy). Twenty female mice were used for the initial screening. The mice that showed the appropriate positioning of the injected cells according to BLI were then divided in two groups: without (MTC vehicle) and with $100 \mathrm{mg} / \mathrm{kg} /$ day bindarit. These treatments started 3 weeks after cell implantation into the mammary fat pads, and were administrated by oral gavage, every day, once a day, for five consecutive days per week, for 2 weeks. The BLI measurements were carried out once a week, and quantified as described above.

\section{Histology and immunohistochemistry}

At the end of each experiment, the mice were sacrificed and their tumor tissues were immediately dissected out and fixed in $4 \%$ paraformaldehyde, for $16-18 \mathrm{~h}$, or frozen in liquid nitrogen. The tissues fixed in $4 \%$ paraformaldehyde were then paraffin embedded, and $7-\mu \mathrm{m}$ sections were prepared for immunohistochemistry staining. Immunoperoxidase staining was performed with the antigen retrieval step, in $10 \mathrm{mM}$ sodium citrate, $\mathrm{pH} 6.0$, for $15 \mathrm{~min}$ at $90{ }^{\circ} \mathrm{C}$.

The sections were incubated with the appropriate primary antibodies, as follows: rabbit anti-CD11b (Abcam) 1:3,000; rat anti-mouse Ly-6G and Ly-6C GR-1 (BD Pharmigen) 1:2,000; rat anti-CD68 (FA-11) (AbCam) 1:100; rabbit anti-CD163 (Santa Cruz Biotechnology, Inc.) 1:500; mouse anti-OPN (Akm2A1) (Santa Cruz Biotechnology, Inc.) 1:1,000; rat anti-CD169 (3D6.112) (AbCam) 1:25; rabbit anti-cleaved Caspase3 (Asp175) (Cell Signaling) 1:500. The negative controls omitted the primary antibody. Sections were revealed using the Liquid DAB + Substrate Chromogen System (Dako), counterstained with Gill's hematoxylin (Bio-Optica) and mounted with Eukitt (Bio-Optica). For statistical analysis, both positive and total cells in three random fields per sections at $\times 40$ magnification were counted and the percent positive cells in each field was calculated from sections obtained from at least four different mice, two treated with bindarit and two treated with MTC (vehicle). Then the averages of the percentages of positive cells in each field per section were calculated and the statistical analysis was performed.

The CD31 immunohistochemistry was performed on frozen sections. Briefly, $7-\mu \mathrm{m}$ sections were prepared from frozen tissue, fixed in pre-cooled acetone $\left(-20^{\circ} \mathrm{C}\right)$ for $10 \mathrm{~min}$, incubated in $0.3 \% \mathrm{H}_{2} \mathrm{O}_{2}$ solution in PBS at room temperature for $15 \mathrm{~min}$, to block endogenous peroxidase activity, blocked and incubated with a rat anti-mouse CD31 
antibody (BD PharMingen) 1:100. The negative controls omitted the primary antibody. Detection was achieved with Liquid DAB + Substrate Chromogen System (Dako), counterstained with Gill's hematoxylin and mounted with Eukit. For statistical analysis, tumors from vehicle-treated $(n=4)$ and bindarit-treated $(n=4)$ mice were analysed. Three sections from each tumor were analysed, $35 \mu \mathrm{m}$ distant from each other. The blood vessels surrounded by CD31-positive cells were counted in five random fields per sections at $\times 20$ magnification. The sum of the blood vessels from the three sections of each tumor was calculated, and statistical analysis was performed.

\section{Western blotting}

Standard methods were used for western blotting. The cells were lysed in lysis buffer and the total protein content was determined by the Bradford method. Tumor tissue specimens were homogenized using a HEIDOLPH Silent Crusher M homogenizer (11-001-596-04-0 probe). The proteins $(50 \mu \mathrm{g})$ were resolved by SDS-PAGE $(10 \%)$ under reducing conditions, and blotted onto polyvinylidene difluoride membranes (Millipore). These membranes were probed with specific antibodies, which were revealed by chemio-luminescence (Amersham Biosciences). The following antibodies were used: rabbit anti-cyclin D1 (92G2) (Cell Signaling) 1:250; rabbit anti-phospho-AKT (S473) (D9E) XP (Cell Signaling Technology) 1:500; rabbit antiIkB- $\alpha$ (Abcam) 1:3,000; rabbit anti-phosphoSmad-3 (Ser423/425) (C25A9) (Cell Signaling Technology) 1:500 and rabbit anti-MCP-1 (FL-148) (Santa Cruz) 1:500. For loading normalization, mouse anti- $\beta$-actin was used (Sigma) 1:10,000.

\section{Statistical analyses}

The statistical comparisons of the data from the in vitro and in vivo treatments were performed using two-tailed students' $t$ tests and ANOVA using StatView software (StatView 5.0.1.0, SAS Institute Inc.), respectively. Statistical significance was established at $p \leq 0.05$.

\section{Results}

Bindarit effects on tumor cell proliferation and migration

To assess the bindarit impairment of cell growth and motility, in vitro cell proliferation and motility assays were performed on PC-3M-Luc2 cells (human prostatic carcinoma) and 4T1-Luc cells (triple-negative mouse breast carcinoma). As previous studies have reported that bindarit selectively inhibits the production of the monocyte chemotactic protein subfamily of inflammatory chemokines (MCP-1, MCP-2, MCP-3) [16], first, the expression levels of the MCP mRNAs was analyzed in these cell lines. As shown in Supplementary Fig. S1, MCP-1 mRNA expression was detected in both cell lines. Very low levels of MCP-2 and MCP-3 mRNA expression was detected in the 4T1-Luc cell line by qRT-PCR. In the PC-3M-Luc2 cell line, MCP-2 and MCP-3 mRNAs expression was not detected. Based on these findings, we focused our attention only on MCP-1.

Bindarit decreased the proliferation of PC-3M-Luc2 cells in dose-dependent and time-dependent manners, as compared to the PBS-treated cells (control) (Fig. 1a). Bindarit significantly reduced the proliferation of PC-3MLuc2 cells at almost all of the experimental points tested (Fig. 1a; $p<0.01$; students' $t$ test). The exception here was 48 -h treatment with 50 and $100 \mu \mathrm{M}$ bindarit, as compared to the PBS-treated cells $(p=0.15$ and $p=0.07$ respectively; students' $t$ test). To unravel the molecular mechanisms involved in this bindarit-induced reduction of cell proliferation, we analyzed cell-cycle perturbation by bindarit. Very mild, but significant, effects were seen on the cell cycle at $24 \mathrm{~h}$ of treatment with 50, 200 and $300 \mu \mathrm{M}$ bindarit, which resulted in a faint increase in the G1-phase cell population, as compared to the PBS-treated cells (Fig. $1 \mathrm{~b} ; p=0.012,0.002$, 0.033 respectively, students' $t$ test). To further address bindarit-induced impairment of cell proliferation, cyclin D1 protein levels, as a marker of the cell cycle, were measured by western blotting. In PC-3M-Luc2 cells, following $24 \mathrm{~h}$ incubation in the presence of bindarit (at both 50 and $100 \mu \mathrm{M}$ ), cyclin D1 levels were reduced (vs. vehicle control) (Fig. 1c). We also analyzed the perturbation of apoptosis caused by this bindarit treatment, using an Annexin V assay. As shown in Fig. 1d, bindarit did not alter the percent cell populations, as compared to PBS-treated cells. These data thus support the concept that the impairment of proliferation observed in the PC-3MLuc2 cell model might be related to an arrest in the G1 phase of the cell cycle. With regard the cell migration, in a modified Boyden-chamber assay that used trans-well technology (see Methods; [27]), 50, 100 and $300 \mu \mathrm{M}$ bindarit significantly impaired PC-3M-Luc2 cell migration, in a dose-dependent manner (Fig. 1e; $p=0.004$, $p<0.0001, p<0.0001$ respectively; students' $t$ test).

Unlike the data obtained in PC-3M-Luc2 cells, at the concentrations tested, bindarit did not impair 4T1-Luc cell proliferation (Fig. 2a), cell cycle (Fig. 2b), apoptosis (Fig. 2c) or cell motility (Fig. 2d). However an inhibitory effect of bindarit on 4T1-Luc cell motility that reached the limit of significance was observed at $300 \mu \mathrm{M}$ bindarit $(p=0.07$, students' $t$ test) (Fig. 2d). 
A

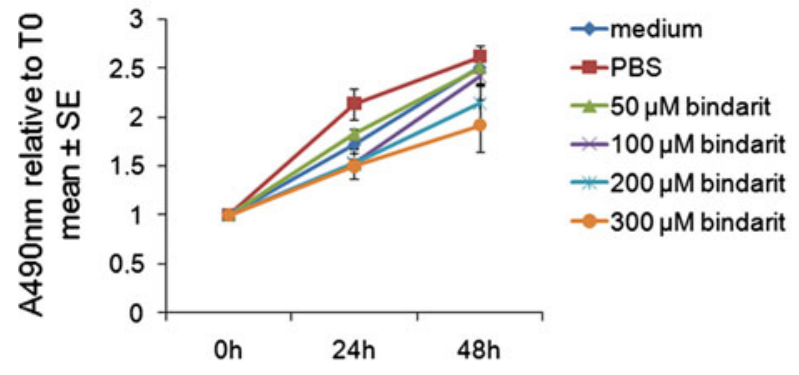

C

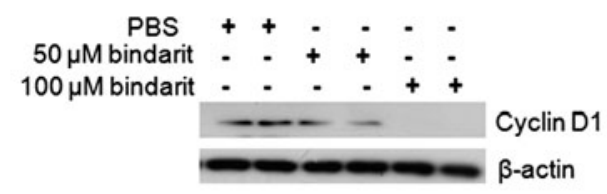

E

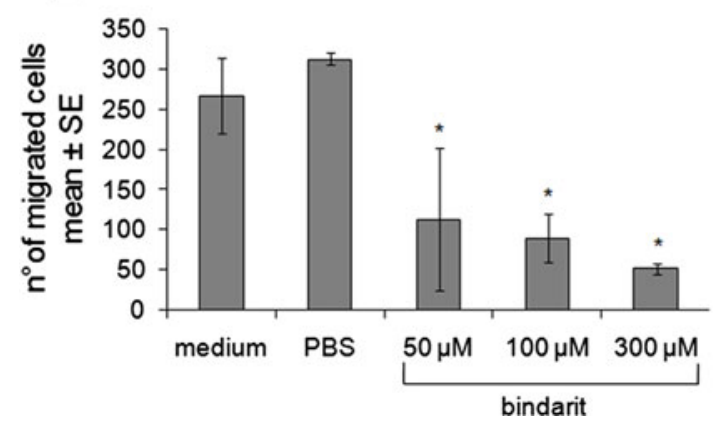

Fig. 1 In-vitro effects of bindarit on PC-3M-Luc2 prostate cancer cells. a Representative cell proliferation assay (of two experiments performed, with similar results) with PC-3M-Luc2 cells treated with PBS (control) or bindarit (as indicated). Data are means $\pm \mathrm{SE}$. $p<0.01$, students' $t$ test for all of the tested experimental points, except 48-h treatment with 50 and $100 \mu \mathrm{M}$ bindarit. b Representative FACS analysis for cell-cycle phases (of two experiments performed, with similar results) of PC-3M-Luc2 cells treated for $24 \mathrm{~h}$ with PBS or bindarit (as indicated). Data are means \pm SE. $* p=0.012$ for $50 \mu \mathrm{M}$ bindarit, $p=0.002$ for $200 \mu \mathrm{M}$ bindarit, $p=0.033$ for $300 \mu \mathrm{M}$ bindarit; students' $t$ test. c Immunoblotting for cyclin D1 in

In vitro effects of immune cells on tumor cell proliferation and motility

In the tumor microenvironment, immune cells produce and secrete MCP-1. To analyze the effects of immune cells on tumor cell proliferation and motility we performed in vitro proliferation and motility assays with 4T1-Luc cells, using the conditioned medium of the murine J774 macrophage cell line. First, using real-time qRT-PCR, we analyzed the expression levels of MCP mRNAs in this cell line. As shown in Supplementary Fig. S1, J774 cells express MCP-1, while very low levels of MCP-2 and MCP-3 mRNAs expression were detected. To further increase the expression of MCP-1 in J774 cells, we stimulated them with LPS. Indeed, previous studies have shown that LPS induces MCP-1 mRNA
B

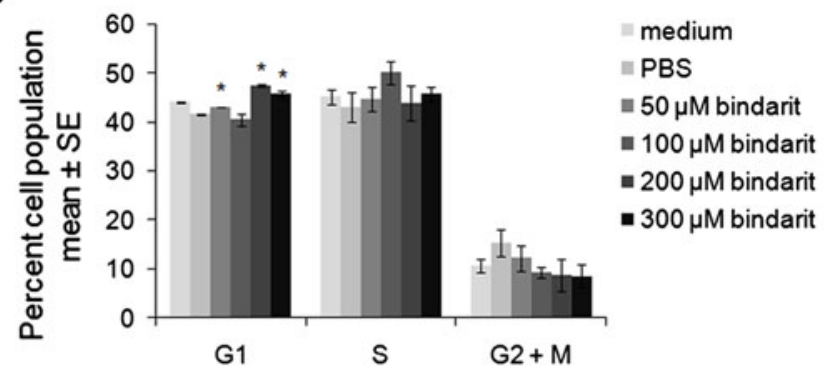

D

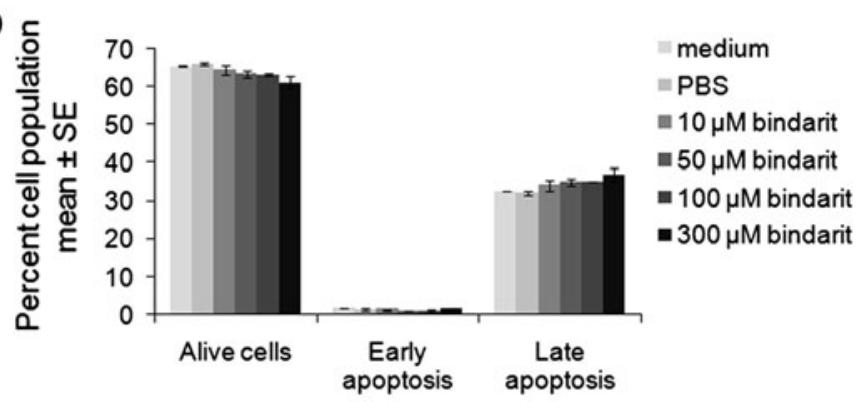

PC-3M-Luc2 cells treated for $24 \mathrm{~h}$ with PBS or bindarit (as indicated). $\beta$-Actin used as control for equal loading. $\mathbf{d}$ Representative FACS analysis for apoptosis evaluation (of two experiments performed, with similar results) of PC-3M-Luc2 cells treated for $24 \mathrm{~h}$ with PBS or bindarit (as indicated). Data are means \pm SE. e Representative motility assays (of two experiments performed, with similar results) in PC-3M-Luc2 cells treated for $4 \mathrm{~h}$ with PBS or bindarit (as indicated). Data are means \pm SE. $* p=0.004$ for $50 \mu \mathrm{M}$ bindarit, $p<0.0001$ for $100 \mu \mathrm{M}$ bindarit, $p<0.0001$ for $300 \mu \mathrm{M}$ bindarit; students' $t$ test

expression and the consequent secretion of protein, and bindarit inhibits this MCP-1 mRNA expression and protein secretion [16, 17]. LPS stimulation of J774 significantly increased MCP-1 mRNA expression ( $p=0.006$; students' $t$ test), which was reduced back to basal levels by bindarit ( $p=0.007$; students' $t$ test) (Fig. 3a). However, bindarit did not alter the basal levels of MCP-1 mRNA expression (Fig. 3a). The 4T1-Luc cell proliferation and motility assays in the presence of LPS-stimulated or unstimulated J774-cell conditioned medium did not change cell proliferation (Fig. 3b) or motility (Fig. 3c). According to these results, the conditioned medium from LPS-stimulated or unstimulated J774 cells treated with $300 \mu \mathrm{M}$ bindarit did not affect 4T1Luc cell proliferation (Fig. 3b) and motility (Fig. 3c). The impairment of cell motility shown in Fig. $3 \mathrm{c}$ for the 

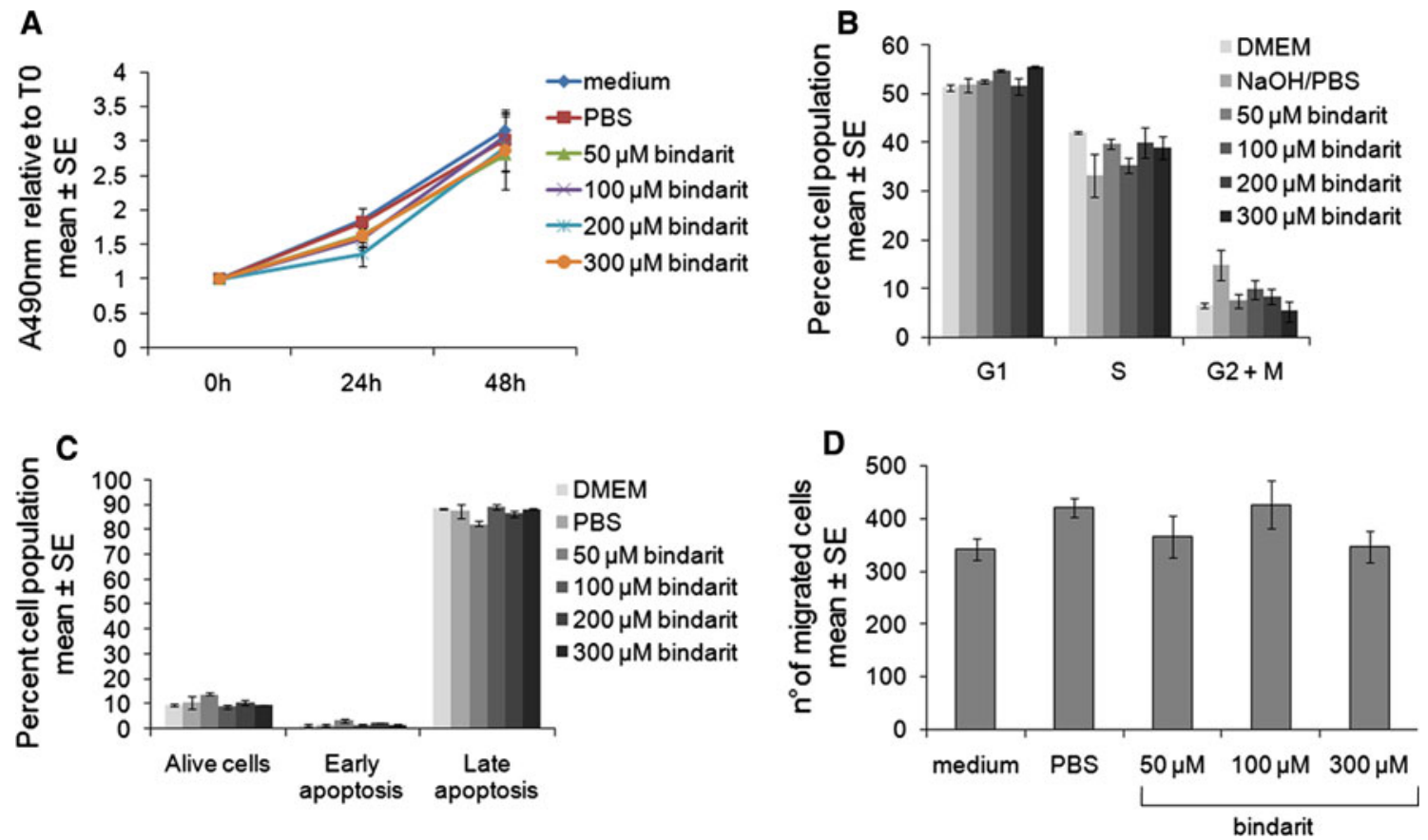

Fig. 2 In-vitro effects of bindarit on 4T1-Luc breast cancer cells. a Representative cell proliferation assay (of two experiments performed, with similar results) with 4T1-Luc cells treated with PBS (control) or bindarit (as indicated). Data are means \pm SE. b Representative FACS analysis for cell-cycle phases (of two experiments performed, with similar results) of 4T1-Luc cells treated for $24 \mathrm{~h}$ with PBS or bindarit (as indicated). Data are means \pm SE. c Representative FACS analysis for apoptosis evaluation (of two experiments performed, with similar results) of 4T1-Luc cells treated for $24 \mathrm{~h}$ with PBS or bindarit (as indicated). Data are means \pm SE. d Representative motility assays (of two experiments performed, with similar results) in 4T1-Luc cells treated for $4 \mathrm{~h}$ with PBS or bindarit (as indicated). Data are means $\pm \mathrm{SE}$ experiments that used conditioned medium from LPS-stimulated J774 cells treated with $300 \mu \mathrm{M}$ bindarit compared to those which used the conditioned medium from LPS-stimulated J774 cells (control), has to be ascribed to direct effects of bindarit on 4T1-Luc cell motility, as illustrated in Fig. 2d.

In summary, in our experimental cell model, macrophages did not affect the tumor cell proliferation and motility.

In vitro signaling pathways alteration by bindarit treatment

To define the effects of bindarit on the molecular signaling pathways that can be altered in cancer cells, and particularly those involved in cell proliferation, inflammation and apoptosis, AKT signaling was investigated, by measuring the phosphorylation status of AKT-1 (on S473). This phosphorylation is a marker of the tumorigenic cascade that leads to cancer metastasis formation [28, 29]. We also measured the level of activation of the NF- $\kappa \mathrm{B}$ signaling pathway (as a measure of the levels of its inhibitor, IkB- $\alpha$ ), as this pathway was found to be an inductor of MCP-1 expression and it was impaired by bindarit [17]. Moreover, we measured the level of activation of the TGF- $\beta$ signaling pathway (as a measure of SMAD-3 phosphorylation status, on Ser423/425), as these are important components in prostate and breast bone niche metastasis formation [30]. Altogether, the results show that all three markers were significantly impaired by bindarit in dose-dependent manners (50 and $100 \mu \mathrm{M})$ in both PC-3M-Luc2 cells (Fig. 4a) and 4T1-Luc cells (Fig. 4b).

Bindarit reduces local tumorigenesis and metastasis formation, and increases animal survival

We tested the anti-metastatic properties of bindarit in vivo in a xenograft mouse model. First, the highly metastatic prostate cancer PC-3M-Luc2 cells were injected intra-cardially into the left ventricle of athymic nude mice. The effects of intraperitoneal administration of vehicle (0.5\% MTC) and bindarit $(100 \mathrm{mg} / \mathrm{kg} /$ day $)$ on metastases growth were monitored through the BLI (Fig. 5a). Treatments were started on day 7 after cell injection, and the experimental protocol was designed for a total length of 12 weeks. As shown Fig. 5a, after 4 weeks treatment, inhibition of the metastatic spread by bindarit reached significance $(p<0.007)$, as compared to 
Fig. 3 In-vitro effects of immune cells on tumor cell proliferation and motility. a MCP-1 gene expression assessed in LPS-stimulated or unstimulated $\mathrm{J} 774$ cells in the presence or absence of bindarit (as indicated). Data are mean values of $2^{-\Delta \Delta C t}$ for MCP-1 \pm SE. $* p=0.006$, $* * p=0.007$; students' $t$ test. b Representative cell proliferation assay (of two experiments performed, with similar results) with 4T1-Luc cells treated with conditioned medium of $\mathrm{J} 774$ cells (as indicated). Data are means \pm SE. c Representative motility assays (of two experiments performed, with similar results) in 4T1-Luc cells treated with conditioned medium of J774 cells (as indicated). Data are means \pm SE. $* p=0.025$, ${ }^{* *} p=0.038$; students' $t$ test
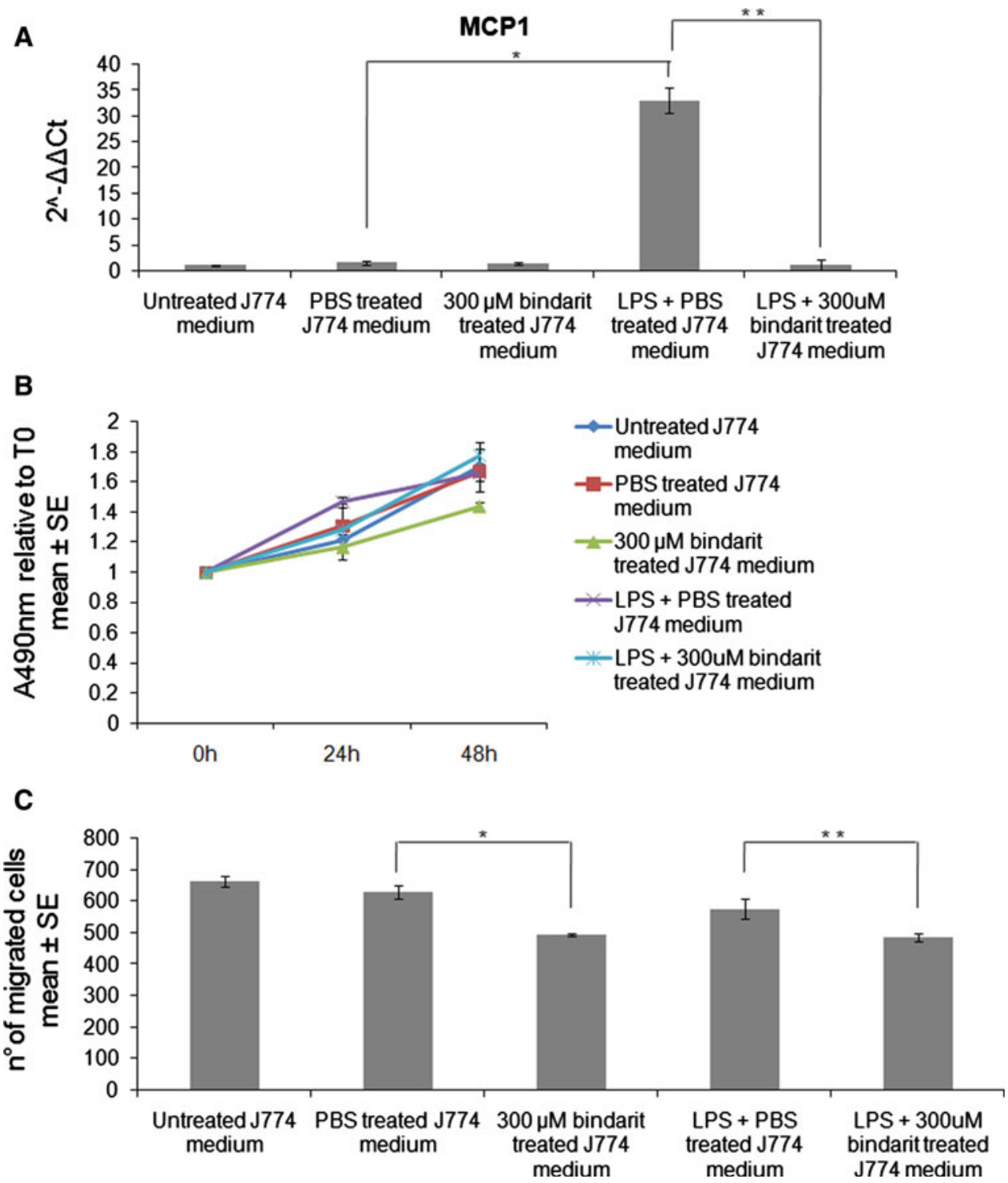

the vehicle-treated mice. Furthermore, as also illustrated in the representative BLI images in Fig. 5a, at 6 weeks bindarit prevented almost all of the metastatic spread $(p<0.002)$. However, in mice which did not show signs of significant metastatic disease (as measured by BLI), the treatment was stopped after 6 weeks. Figure 5b shows the mice treated after 6 weeks and their survival. Figure 5c shows representative images of bindarit-treated mice after 6 and 12 weeks. Indeed, survival curve analysis demonstrated significant inhibition $(p<0.0001)$ by bindarit over the vehicle-treated mice: at 84 days post-injection, all of the vehicle treated mice were dead, while $64 \%$ of the mice treated with bindarit were still alive (Supplementary Fig. S2 and Table S1). Moreover, when the trial was stopped at 120 days post injection, $36 \%$ of the mice in the bindarit-treated group was still alive and free of metastatic sites, as demonstrated by BLI scanning (Tables S1 and S2).
Bindarit was further investigated in an in vivo 4T1-Luc breast-cancer xenograft mouse model. To determine the effects of bindarit on primary tumor growth, the 4T1-Luc cells were orthotopically implanted into the mammary fat pads of Balb/c syngeneic mice. The cells were allowed to grow over 3 weeks. Then, the mice started to receive oral vehicle $(0.5 \%$ MTC) or bindarit $(100 \mathrm{mg} / \mathrm{kg} / \mathrm{day})$, with treatments that lasted for 2 weeks. Tumor growth was monitored by BLI. The statistical analysis of data showed bindarit significantly inhibited $(p=0.02)$ local tumorigenesis, with a $50 \%$ reduction in tumor growth, as compared to the vehicle-treated mice (Fig. 6a and Supplementary Table S3). Representative images of vehicle and bindarit treated mice are shown in Fig. 6 b.

Altogether, these data demonstrate that bindarit can inhibit both in vivo metastasis formation and primary tumor growth. 


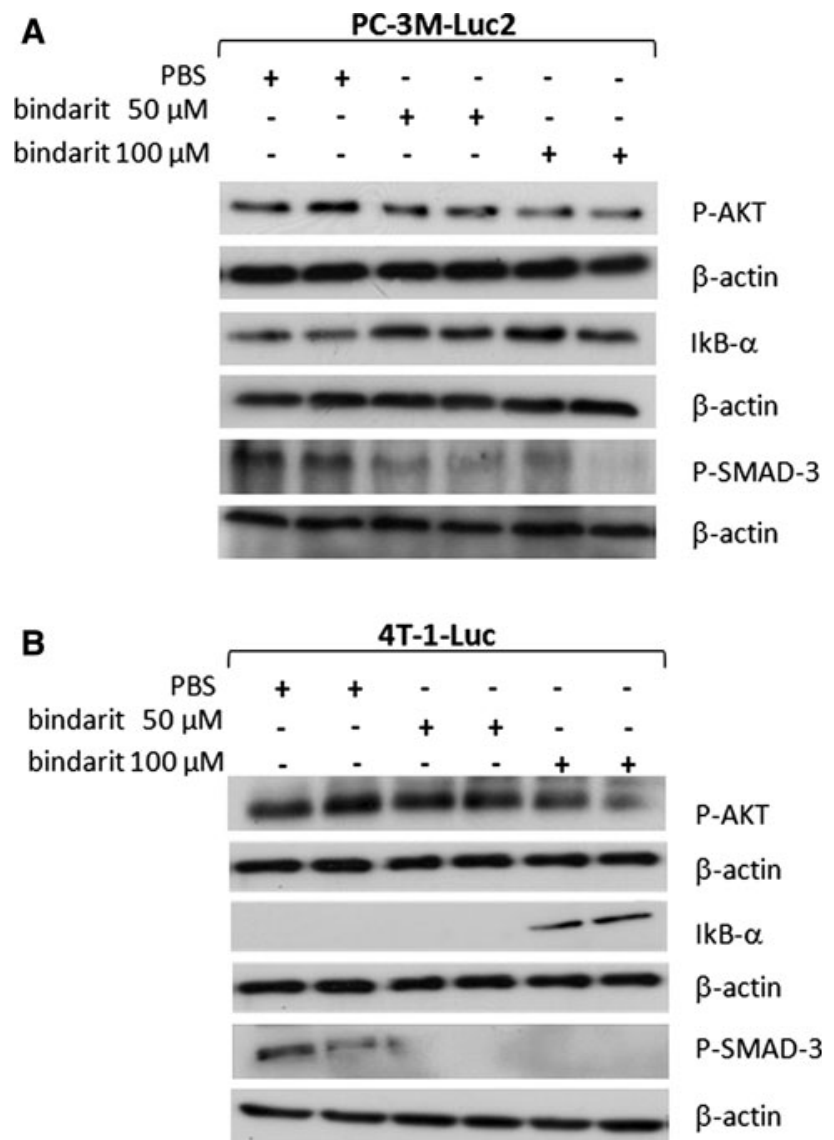

Fig. 4 Representative western blotting of a PC-3M-Luc2 and b 4T1Luc cells at $4 \mathrm{~h}$ treatment with vehicle (PBS) or with 50 and $100 \mu \mathrm{M}$ bindarit. Reduced expression of phosphorylated AKT and phosphorylated SMAD, and the enhanced expression of IkB- $\alpha$ were observed. $\beta$-Actin was used for loading normalization

Bindarit reduces monocyte infiltration into tumors and tumor angiogenesis

To achieve metastasis formation, tumorigenic cells need to communicate with their microenvironment, which includes the cells of the immune system. To determine the effects of bindarit treatment on immune-cell infiltration into the tumor microenvironment, immunohistochemistry analyses of TAMs and MDSCs were conducted on sections of primary tumors from the breast cancer xenograft mice that had 4T1-Luc cells implanted into the mammary fat-pad, and were treated with vehicle (PBS) or $100 \mathrm{mg} / \mathrm{kg} / \mathrm{day}$ bindarit (5 days/week). In the tumors from the vehicletreated mice, hematoxylin/eosin staining showed compact and rounded shapes, as characteristic of very dense cell aggregates (Fig. 6c). In contrast, tumors from bindarittreated mice showed a less compact structure, due to large regions of necrosis (Fig. 6c, arrows). It is worth noting that tumors from bindarit-treated mice showed a reduction in the expression of the MCP-1 protein and enhanced expression of the NF- $\kappa$ B inhibitor IkB- $\alpha$, as detected by western blotting (Fig. 6d).

With regards to local infiltration by inflammatory cells, tumors from mice treated with bindarit showed a significant reduction in the number of MDSCs (CD11b and GR-1 positive cells), as compared to the vehicle-treated mice (Fig. 7a, b; $p=0.0001$ for both). Similarly, tumors from mice treated with bindarit showed a significant reduction in the number of TAMs (CD68, CD163 and CD169 positive cells), as compared to the vehicle-treated mice (Fig. 7a, b; $p<0.0001$ for all of the markers).

We also looked at a typical marker of the 'metastatic niche': osteopontin (OPN), a protein that is involved in cell adhesion, chemoattraction and immunomodulation. OPN expression was decreased in the tumors from bindarittreated mice, as compared to the vehicle-treated mice (Fig. 7a, b; $p<0.0001$ ). Interestingly, tumors from the bindarit-treated mice showed OPN staining localized to the inner part of the tumor (Fig. 7a).

Activation of caspase-3 in tumor specimens was also evaluated. Bindarit treatment did not induce apoptosis, as compared to the vehicle-treated mice (Supplementary Fig. S3).

Previous studies have suggested an important role for MCP-1 in tumor vascularization. Indeed, these studies demonstrated that tumors from nude mice subcutaneously injected with MCP-1-overexpressing melanoma cells show strong positivity for CD31 (a marker of endothelial cells) and a massive branched intratumoral vascular network. This vascularization was completely absent in control tumors [26]. Moreover, these studies also showed that in vitro, MCP-1 induced the organization of endothelial cells into tubular structures, and treatment with a specific antibody against MCP-1 prevented the endothelial cell organization into tubules [26]. To assess the ability of bindarit to impair in vivo tumor angiogenesis in our breast cancer mouse model, immunohistochemistry analyses of endothelial cells (CD31-positive cells) were conducted on sections of 4T1-Luc primary tumors. No differences were observed in the staining intensities for CD31 in the tumors from bindarit-treated mice compared to vehicle-treated mice. However while the tumors from vehicle-treated mice showed CD31 staining that delimited circular structures, representing blood vessels, the tumors from bindarit-treated mice showed CD31-positive cell clusters that did not delimit circular structures (Fig. 8a). Statistical analysis showed that bindarit statistically reduced the number of blood vessels compared to that in vehicle-treated mice (Fig. 8b; $p<0.0001$ ).

In summary, our data show that in vivo pharmacological inhibition of MCP-1 by bindarit impairs the infiltration of immune cells into the tumor microenvironment and the formation of new blood vessels. 

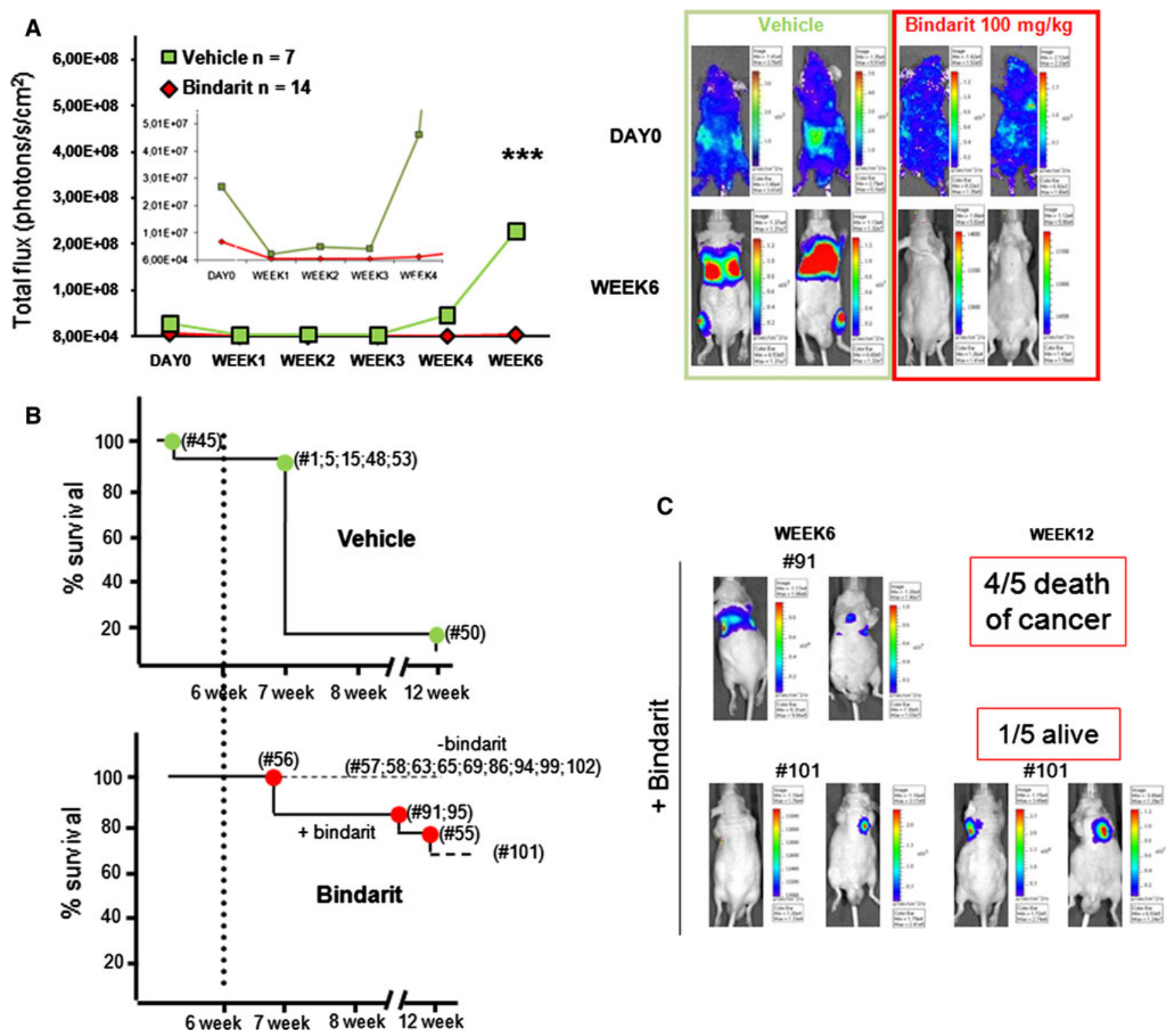

Fig. 5 Bindarit protection against metastatic foci development following intra-cardial injection of highly metastatic prostate cancer PC-3M-Luc2 cells into the left ventricle of athymic nude mice. a Left metastasis growth curves established by in vivo BLI over 6 weeks of treatment. Bindarit significantly reduced metastasis development after 4 weeks of treatment $(p<0.007)$, with a further significantly enhanced effect at 6 weeks $(* * * p<0.002)$. Right representative BLI images of vehicle-treated and bindarit-treated $(100 \mathrm{mg} / \mathrm{kg} / \mathrm{day}$,

\section{Discussion}

In recent years, cancer research has focused on the mechanisms that sustain metastasis formation and progression, thus investigating more deeply the relations between cancer and inflammation [31]. In particular, dysregulation of chemokines and chemokine receptors has been demonstrated to alter tumor development and progression, through enhanced cell proliferation and increased cell

intraperitoneal administration) mice, at day 0 and week 6 of treatment. The inner panel is a magnification of curves from day 0 to week 4. b Survival curves of vehicle-treated and bindarit-treated mice after 6 weeks of treatment. In mice not showing bioluminescence after 6 weeks, the treatment was stopped and metastasis relapse was analyzed. $\mathbf{c}$ Representative BLI images of bindarit-treated mice at 6 and 12 weeks treatment

invasiveness, and through increased angiogenesis and the recruitment of immune cells that promote tumor growth [32]. Chemokines are considered as a second level of communication between tumor cells and their microenvironment, and significant production of chemokines has been seen, as mostly expressed by cancer-associated fibroblasts, mesenchymal stem cells, endothelial cells, TAMs, and tumor-associated neutrophils [33]. Chemokines might be expected to have either growth-promoting or 

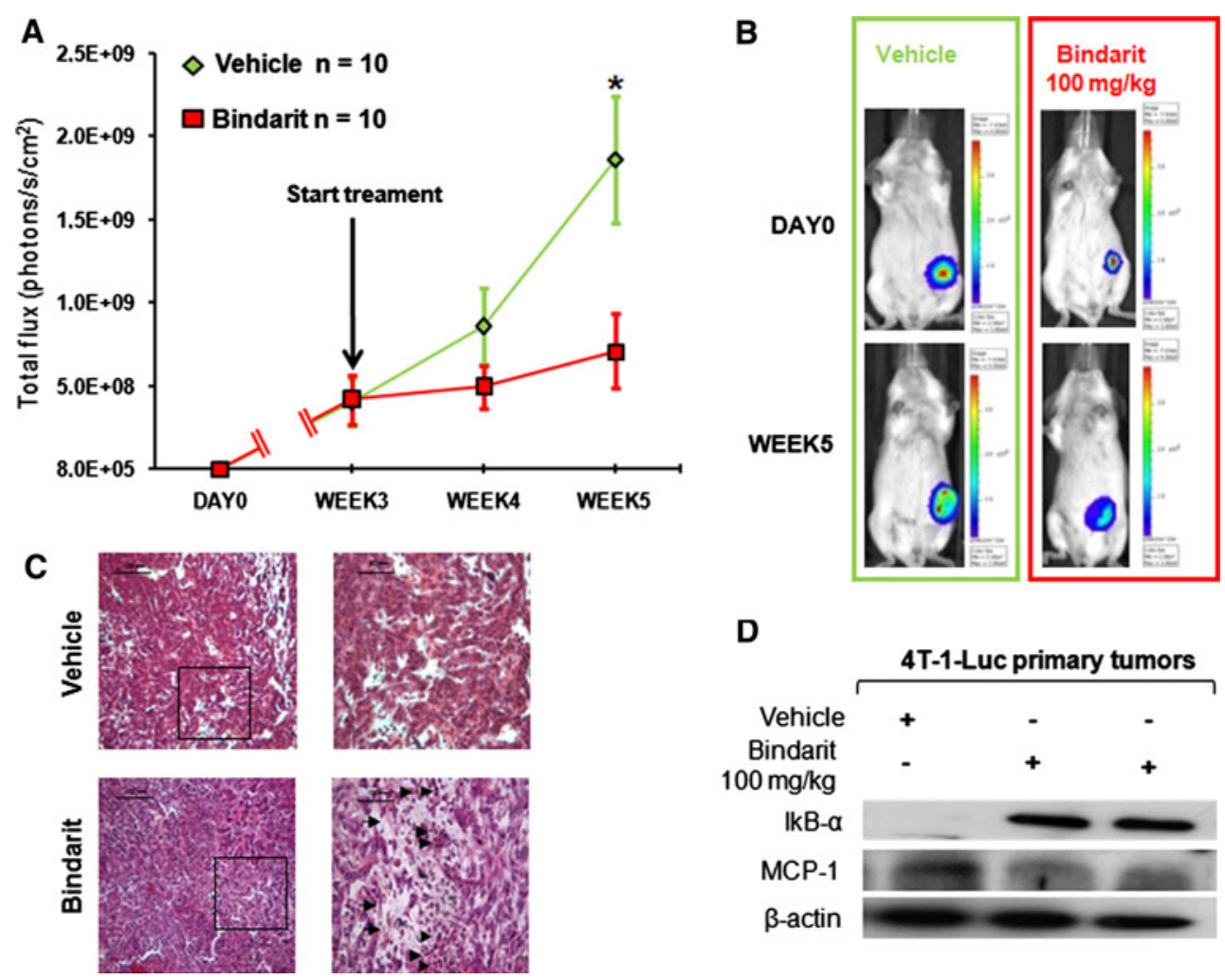

Fig. 6 Bindarit effects on local tumorigenesis. a Time-course of BLI signals from 4T1-Luc tumor cells implanted into mouse mammary fat pad at day 0 , followed by oral gavage administered treatments with vehicle and $100 \mathrm{mg} / \mathrm{kg} /$ day bindarit from week 3. Data are total flux means \pm SE. Bindarit significantly $(* p<0.02$; ANOVA $)$ reduced tumor growth following 5 weeks of implantation and over 2 weeks of treatment. b Representative BLI images of vehicle-treated and bindarit-treated $(100 \mathrm{mg} / \mathrm{kg} / \mathrm{day}$, oral gavage administration) mice, at day 0 and after 2 weeks of treatment (week 5 following implantation; see a). c Hematoxylin/eosin staining of tumor samples obtained from mice treated as in (a). Left $\times 5$ magnification, right $\times 40$ magnification of the black rectangles in the corresponding left panels. Arrowheads necrosis regions. d Representative western blotting showing a decrease in MCP-1 expression and an increase in IkB- $\alpha$ expression upon bindarit treatment in tumors obtained from mice treated as in (a). $\beta$-Actin was used for loading normalization growth-inhibiting influences on cancer initiation, which would depend on the particular environmental settings in which they are expressed. Within their circuit of communication, MCP-1 (CCL2) is a potent chemoattractant for monocytes, memory $\mathrm{T}$ lymphocytes, MDSCs and natural killer cells. MCP-1 expression has also been demonstrated in a wide variety of cancer types, where it appears to stimulate vascularity, and cell proliferation and migration through direct mechanisms, and also indirectly via TAM recruitment [15, 26, 34-37]. In addition, activation of the MCP-1/CCR2 axis promotes cell invasion in prostate cancer and tumor-induced bone lesions. On the other hand, in the presence of human metastatic breast cancer cells, osteoblasts undergo an inflammatory stress response, which includes the production of MCP-1 [6, 38-40].

In breast cancer, MCP-1 is expressed and associated with the formation of bone-marrow metastases $[6,8]$, and its co-expression with the other $\mathrm{C}-\mathrm{C}$ chemokine RANTES is associated with progression of disease in the advanced stages [41]. In prostate cancer, MCP-1 also acts as a paracrine and autocrine factor for growth and invasion [42], and for bone metastasis [43, 44]. The MCP-1 CCR2 axis contributes to the development and progression of cancer diseases through two major mechanisms: autocrine effects and paracrine effects. The autocrine effects of MCP-1 on malignant cells that produce MCP-1 and also express its receptor, CCR2, promote the survival and growth of $\mathrm{CCR}^{+}$cancer cells. As a paracrine factor, MCP-1 promotes the attraction of bone-marrow-derived monocytic cells from the bone marrow to target tissues by chemotaxis. These cells accumulate at the tumor site, to initially support tumor development and angiogenesis, resulting in enhanced levels of various inflammatory cytokines, growth factors and chemokines, which further contribute to tumor development and progression. Moreover, as a paracrine factor, MCP-1 induces the differentiation of bone-marrow-derived monocytic cells into TAMs, and shows pro-angiogenic effects on endothelia that also expresses CCR2 [45]. Moreover, in PC-3M-Luc2 cells, MCP-1 can protect the cells from autophagic death via the 
A 4T-1-Luc primary tumors
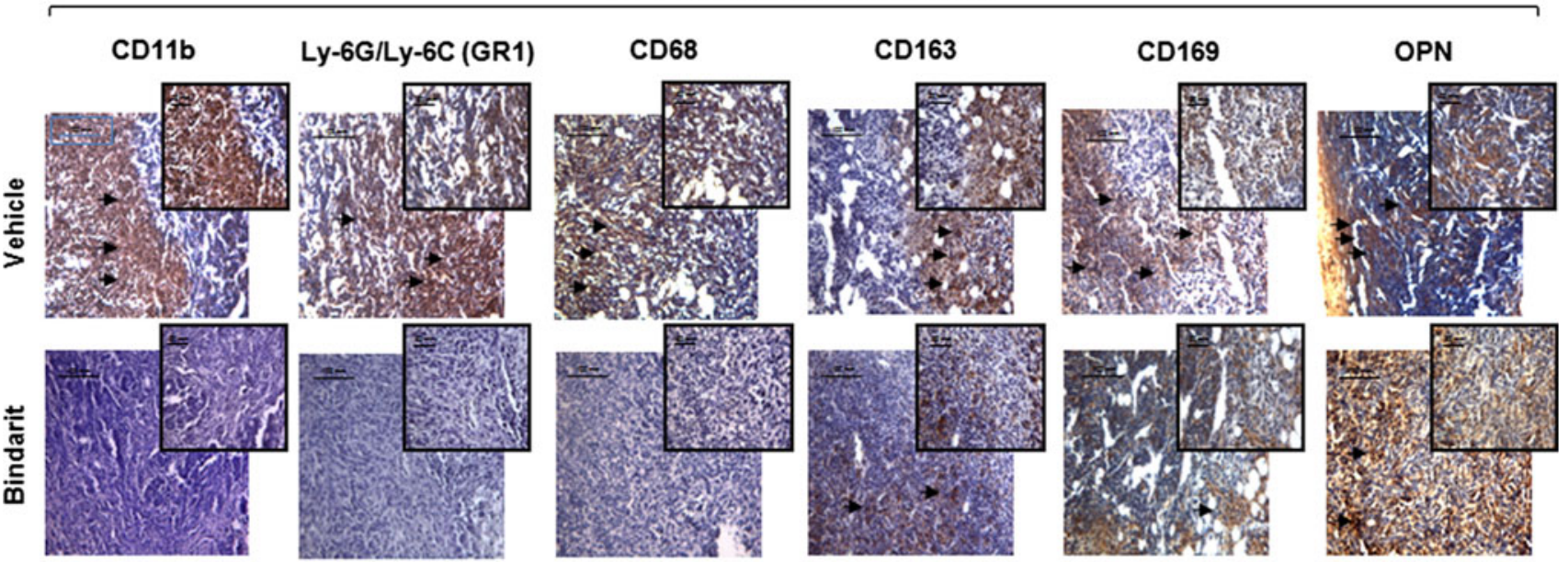

B
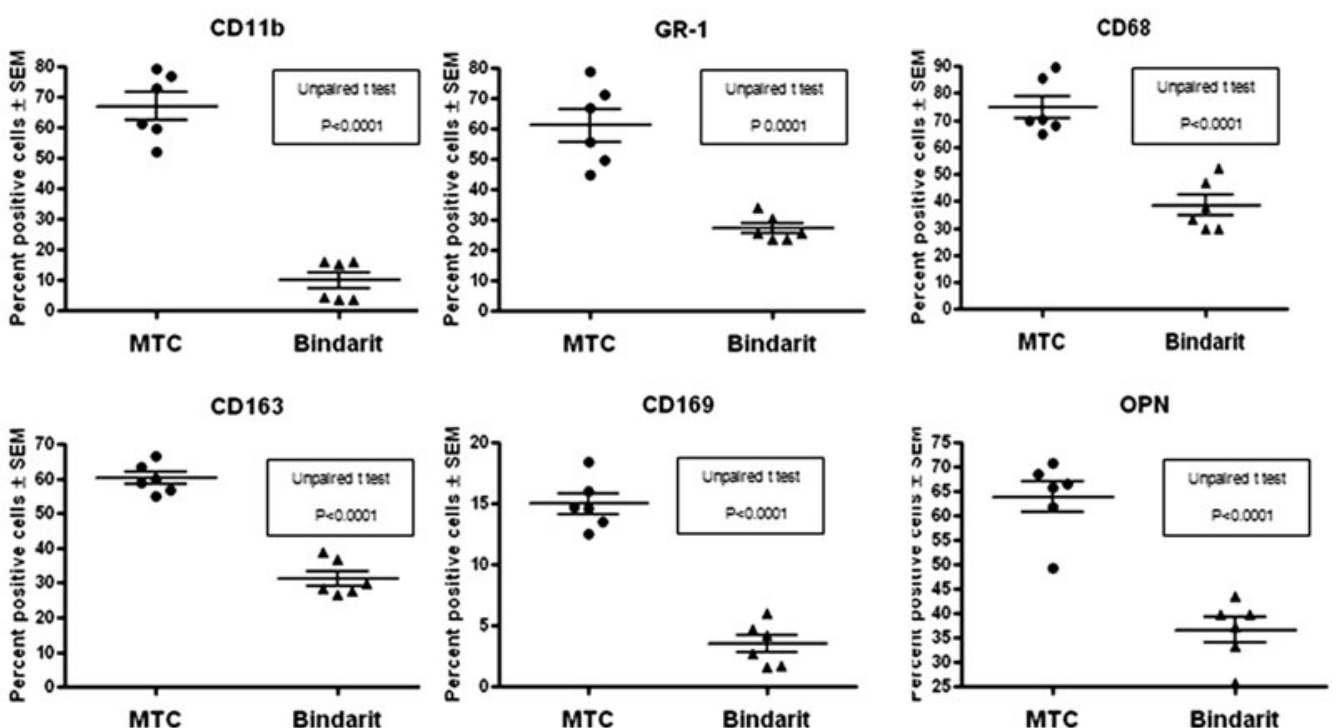

Fig. 7 Bindarit effects on TAMs, MDSCs and metastatic niche markers in tumors formed following mammary gland implantation of highly metastatic breast cancer 4T1-Luc cells injected under the left femoral mammary gland in syngeneic Balb/c mice. a Representative images of immunohistochemistry staining for $C D 11 b$ (MDSC marker), GR1 (MDSC marker), CD68 (TAM marker), CD163 (TAM marker), CD169 (TAM marker) and $O P N$ (immunomodulating

phosphoinositide 3-kinase/AKT/survivin pathway, and it induces transendothelial migration of these cells via activation of the small GTPase Rac [28, 29, 46].

In both prostate and breast cancer, neutralizing antibodies to MCP-1 have been monitored in preclinical mouse studies. In the in vitro PC-3M-Luc2 cell model of prostate cancer, parathyroid-hormone-receptor-induced MCP-1 transcription promotes osteoclast differentiation, proliferation and invasion. This induction was partially inhibited by an MCP-1-neutralizing antibody [47]. In addition, it has been shown that MCP-1 is involved in possible resistance to docetaxel [48]. Recent studies have described proof-ofconcept animal models with the use of human or mouse chemokine). The arrowheads positives cells (MDSCs and TAMs) within the staining. b Quantification of samples as in (a), as counts of percent of positive cells for staining of indicated proteins. Each data point represents the mean of the counts from three fields of each $50 \mu \mathrm{m}$ consecutive sections ( \pm SEM lie within each data point) from two different tumors. $p<0.0001$; all of the data

anti-MCP-1 antibodies, or combinations of both, to block PC-3M-Luc2 prostate cancer and 4T1-Luc breast cancer cells, which are known to develop metastases [6, 11, 49, 50]. These antibody treatments end with the inhibition of prostate tumor growth and tumor cell migration in vivo through direct effects on prostate cancer cells and a block of TAM infiltration into the tumors (most probably through an indirect effect). Some of these antibodies are currently being tested in humans (see website: "Clinical trials at http://clinicaltrials.gov/"). However, while anti-MCP-1 antibody treatment has been demonstrated in mice to reduce tumor burden, it did not completely block primary tumor and metastases formation. Of note, Qian et al. [13] 

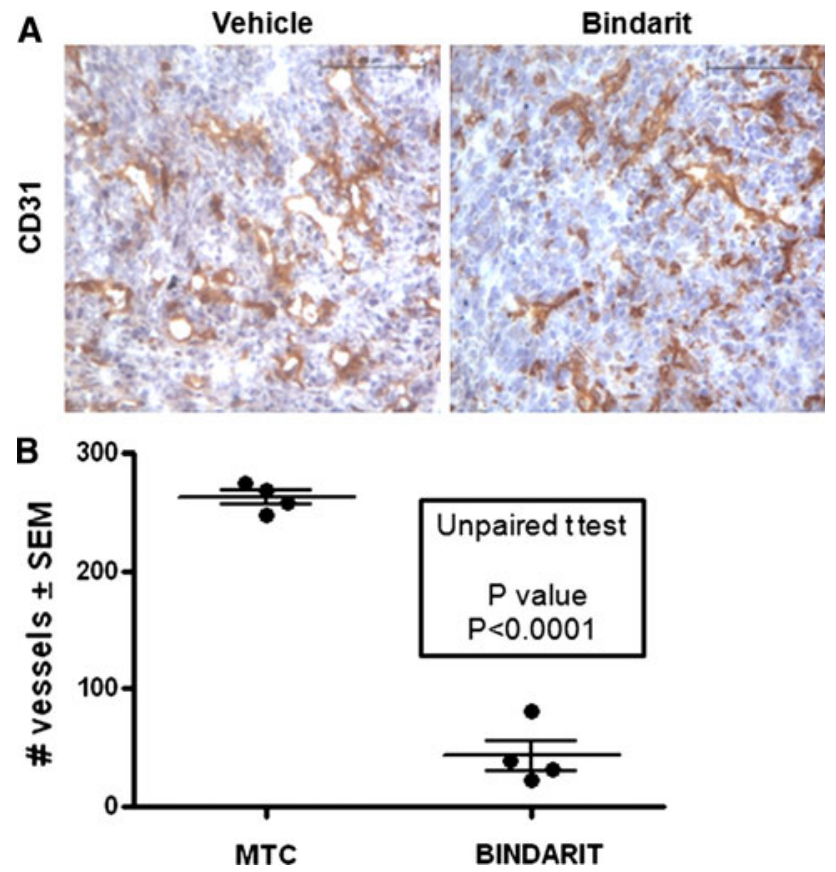

Fig. 8 Bindarit impairs angiogenesis. a Representative images of CD31 immunohistochemistry in sections of 4T1-Luc primary tumors from mice oral gavage administered with vehicle or bindarit. b Numbers of blood vessels, fully surrounded by CD31-positive cells, in sections of 4T1-Luc primary tumors as for (a). Data are means \pm SEM. $p<0.0001$ (ANOVA)

strengthened the importance of a therapeutic approach that targets monocyte recruitment in lung metastasis assays of breast cancer: the use of human and mouse anti-MCP-1 antibodies either alone or in combination inhibited spontaneous lung metastases forming from orthotopically implanted MDA-MB-231 cells, and prolonged the survival of the mice. This result thus indicates the importance of continuous recruitment of inflammatory monocytes and their differentiation into TAMs. In support of this hypothesis, other studies have shown that an anti-MCP-1 antibody cannot reduce liver metastasis of Met-1 cells, which normally do not recruit inflammatory monocytes [13].

These studies underline the important role played by MCP-1/CCL2 in malignant progression and metastasis formation of tumors, and suggest that this chemokine is a useful therapeutic target in cancer treatment. In light of these data, we here assessed the clinical potential of bindarit in cancer treatment, which is a small molecule identified as an inhibitor of the MCP subfamily of proinflammatory chemokines, [21, 23, 51-53]. Gazzaniga et al. [26] provided the first supporting evidence for the use of bindarit in a tumor model. Indeed, they demonstrated that bindarit can inhibit melanoma growth and macrophage recruitment [26]. In our study, we further emphasize the ability of bindarit to inhibit prostate and breast cancer in proof-of-concept animal models and provide insights into the molecular mechanisms of its action. In vitro, bindarit can impair the proliferation and motility only of the PC3M-Luc2 cells; indeed, no effects were observed on the proliferation and motility of 4T1-Luc cells, probably due to the higher MCP-1 expression levels in this cell line not sufficiently being inhibited by the $300 \mu \mathrm{M}$ concentration dose (see Figs. S1 and Fig. 2d), as compared to PC-3MLuc2 cells. Nevertheless, in vivo studies, bindarit efficiently impaired both metastasis growth in the PC-3MLuc2 prostate cancer xenograft mouse model and primary tumor growth in the 4T1-Luc breast cancer xenograft mouse model (Fig. 5). These results have to be ascribed to the ability of bindarit to impair the autocrine and paracrine effects of MCP-1, thus affecting tumor growth both directly and indirectly, through inhibition of TAM and MDSC recruitment and blood vessels organization in the tumor microenvironment, thus resulting in increased survival of bindarit-treated mice compared to vehicle-treated mice. There is some evidence now that MCP-1 has a role both in transendothelial migration [47] and in the creation of the fertile environment in bone for metastasis formation, with these tumorigenic cells expressing CCR2 as well as having elevated levels of MCP-1. This will promote an autocrine/paracrine loop [11, 54] and attract TAMs [13]. Based on these findings, the bindarit therapeutic efficacy to impair metastasis is probably due to its ability both to interfere with cancer cell extravasation and to inhibit the creation of the fertile environment in metastatic sites.

MCP-1/CCR2 has been previously and successfully targeted using gene transfer, an MCP-1-neutralizing antibody, or a CCR2 antagonist in rodent models [11, 14]. However, most of these approaches are too invasive or impractical for use in large animals or humans. Furthermore, to date, their functional consequences remained unclear, thus supporting the economic and physiological advantages for the use of specific small molecules.

Importantly, preclinical studies demonstrated that bindarit has a safe toxicological profile, with an in vivo half-life of about $10 \mathrm{~h}$; it is also devoid of immunosuppressive, mutagenic, and carcinogenic effects. Phase I clinical studies have demonstrated that bindarit (up to a dose of $1,200 \mathrm{mg}$ BID) is well tolerated and confirmed the lack of overt toxicity suggested by preclinical studies (Product Data Sheet, Angelini Research Centre). Results of Phase II clinical studies confirmed the good tolerability profile of bindarit and demonstrated significant effects in patients with kidney disease [20]. This would represent a clear advantage for future clinical trials that are designed to determine the potential of small molecules that can inhibit MCP-1 in prostate and breast cancer.

Xenografts using 4T-1 murine breast carcinoma cells in a 4T-1 syngeneic strain of Balb/c mice are considered to be 
a more predictive in vivo system with respect to xenografts using human tumor cells in immunodeficient mice, as this animal model investigates tumor-cell behavior in a complete and intact immune system. Treatment with bindarit in this syngeneic mouse model system can differentiate vehicle treatment from active treatment through differential expression of TAM and MDSC markers, and through decreased metastatic niche markers, such as OPN $[55,56]$. Elevated levels of OPN are associated with a variety of pathological processes, including cancers. Indeed, OPN can control tumor cell motility and invasion through engagement of the CD44 receptors, and it is highly expressed by TAMs [57]. Also, CD11b ${ }^{+} \mathrm{Gr}^{+}$MDSCs are important contributors to tumor progression, as they can secrete immunosuppressive mediators, induce T-lymphocyte dysfunction, and mediate tumor resistance to antivascular endothelial growth factor therapy [30, 58-60].

Our analysis of the pan-monocytes/macrophages marker CD68 [61], the mature tissue/residential macrophages marker CD163 [61], and the activated macrophage marker CD169 [61] demonstrated regulation of their expression by bindarit in vivo. It is known that these markers are regulated by chemokines that can promote monocyte differentiation to a distinct type of macrophage. CD163 is a pathogen recognition receptor and its expression is enhanced by the chemokine CXCL12. This induces secretion of angiogenic factors, and it has been found in peri-vascular sites of tumors [62-65]. A recent report showed that in the lymph nodes, macrophages expressing CD169 (or sialoadhesin) can take up dead tumor cells, and can directly cross-present tumor antigens to cytotoxic $\mathrm{T}$ cells. Mice lacking $\mathrm{CD} 169^{+}$macrophages at the time of induced tumor degradation fail to induce antitumor immunity [66]. In our in vivo assay, in bindarit-treated animals, we found preferential staining of these markers on the inner site of the tumor, closest to the tumor sections with necrosis activation, thus potentially enhancing this recently described phenomena. Future investigations need to more deeply address this additional action of bindarit.

To date, the expression and role of each of these macrophage markers are still a matter of debate $[62,63,65,67$, 68]. Indeed, within the migration, proliferation, remodeling and angiogenesis processes, the presence of infiltrating white blood cells and chemokines and cytokines is a hallmark of cancer-associated inflammation. While classically fully activated macrophages (type M1) can elicit tumordestructive reactions [69], TAMs or differentially activated macrophages (type M2) can promote tumor growth. Interestingly, the annexin $\mathrm{V}$ apoptotic marker was found not to be activated during bindarit treatment, thus excluding an apoptotic process in vitro. This result was also confirmed in vivo by immunohistochemical experiments for cleaved caspase-3. In our cellular models, bindarit decreased activation of the PI3K/AKT signaling pathway through down-regulation of AKT phosphorylation. It is well known that MCP-1 elicits a strong survival advantage in prostate cancer PC-3 cells through this pathway-dependent regulation of autophagy via the mammalian target of rapamycin pathway and increased survivin expression [28, 29]. These data support our results relating to the lack of apoptosis activation by bindarit, and they suggest that further studies have to be performed to address the regulation of autophagy by bindarit.

On the other hand, our cell-cycle analysis of bindarittreated cells showed a slight block in the G1 phase of the cell cycle, and western blotting using an anti-cyclin D1 antibody revealed a decrease in its expression after bindarit treatment. As it is known, cyclin D1 promotes progression through the G1-S phase of the cell cycle, and amplification or over-expression of cyclin D1 is predominantly associated with human tumorigenesis and cell metastases. This gives cyclin D1 a pivotal role in the development of a subset of human cancers, which include parathyroid adenoma, breast cancer, colon cancer, lymphoma, melanoma, and prostate cancer [70, 71]. Moreover, cyclin D1 has been associated with functions that are cell-cycle independent, as cyclin D1-deficient mice are resistant to oncogeneinduced malignancies, and migration of cyclin $\mathrm{Dl}^{-/-}$bonemarrow-derived macrophages is reduced. Thus, apart from the proliferative and motility defects in the tumor cells themselves, reduced motility and invasiveness of cyclin $D 1^{-1-}$ TAMs might contribute to the tumor resistance of these mice [72]. The data presented here lead us to the hypothesis that bindarit can act against protein kinases that are involved in the control of the cell cycle. This hypothesis will be an issue of our future investigations.

In conclusion, bindarit can inhibit the proliferation and migration of cancer cells in vitro. This effect is not due to programmed apoptotic cell death, but most probably to a slower proliferation rate and an inhibition of the migration rate. Also, in vivo, in these metastatic (prostatic carcinoma) and local (breast carcinoma) tumorigenesis mouse models, bindarit inhibited tumor growth and metastasis formation, and prolonged the survival of the mice. We can speculate at this time that by inhibiting both cyclin D1 and MCP-1, bindarit indirectly regulates the balance between the M2/TAMs versus M1 types of macrophages, which results in reduced tumorigenesis.

Using structure-activity relationships and designed chemical synthesis, we have further identified in our laboratory new compounds with higher inhibition potential of MCP-1 in vitro. These molecules will be used in future studies to improve and enhance M1-type macrophage activation during treatment of metastatic cancers.

Finally, this study adds a new small molecule to the list of cancer-therapeutics that impair tumor growth and 
metastasis by targeting inflammation. This action of bindarit specifically targets MCP-1, thus down-regulating the pro-tumor activity of MCP-1. As this bindarit activity can completely block tumor growth and metastasis, bindarit can be used as a single agent. However, while bindarit administration in humans does not raise specific concerns, we do not know at this time how long the activity of bindarit will be sustained in the metastatic niche. Additional studies and clinical trials will be required to refine any proposed dose schedule for the use of bindarit in the treatment of cancers. Moreover, we cannot at present exclude roles for the other MCP family members in tumor formation, whereby, for example, in mouse, MCP-3 has a key role in the egress process of cells from bone marrow [73]. In conclusion a combination of bindarit with currently available anti-cancer agents might represent the therapy of the future for the treatment of metastatic cancers.

Acknowledgments We thank the Core Services platforms of CEINGE Laboratories, and Prof. Luigi del Vecchio, Head of the Cell Sorter Facility, and its facility for helpful and critical discussions. This study was funded by Angelini Grants ACRAF 004FA09369, 004FA09383 (MZ) and the European GRANT-FP7-Tumic HEALTHF2-2008-201662 (MZ). VDD was supported by Fondazione San Paolo (IM) and Tumic EU-FP7, DS was supported by the Dipartimento di Biochimica e Biotecnologie Mediche, 'Federico II' University of Naples, DMD was supported by Dottorato in Medicina Molecolare e Genetica, 'Federico II' University of Naples, and LL was supported by Scuola di Specializzazione in Genetica Medica, 'Federico II' University of Naples, Italy.

\section{References}

1. Havens AM et al (2008) An in vivo mouse model for human prostate cancer metastasis. Neoplasia 10(4):371-380

2. Spano D, Zollo M (2012) Tumor microenvironment: a main actor in the metastasis process. Clin Exp Metastasis 29(4):381-395

3. Pikarsky E et al (2004) NF-kappaB functions as a tumour promoter in inflammation-associated cancer. Nature 431(7007):461-466

4. Mizutani K et al (2009) The chemokine CCL2 increases prostate tumor growth and bone metastasis through macrophage and osteoclast recruitment. Neoplasia 11(11):1235-1242

5. Aggarwal MM et al (2010) Measurement of the bottom quark contribution to nonphotonic electron production in $\mathrm{p}+\mathrm{p}$ collisions at sqrt [s] $=200 \mathrm{GeV}$. Phys Rev Lett 105(20):202301

6. Lu X, Kang Y (2009) Chemokine (C-C motif) ligand 2 engages CCR2 + stromal cells of monocytic origin to promote breast cancer metastasis to lung and bone. J Biol Chem 284(42): 29087-29096

7. Zhang J, Lu Y, Pienta KJ (2010) Multiple roles of chemokine (C$\mathrm{C}$ motif) ligand 2 in promoting prostate cancer growth. J Natl Cancer Inst 102(8):522-528

8. Takahashi M et al (2009) Chemokine CCL2/MCP-1 negatively regulates metastasis in a highly bone marrow-metastatic mouse breast cancer model. Clin Exp Metastasis 26(7):817-828

9. Nam JS et al (2006) Chemokine (C-C motif) ligand 2 mediates the prometastatic effect of dysadherin in human breast cancer cells. Cancer Res 66(14):7176-7184
10. Zhang J, Patel L, Pienta KJ (2010) CC chemokine ligand 2 (CCL2) promotes prostate cancer tumorigenesis and metastasis. Cytokine Growth Factor Rev 21(1):41-48

11. Loberg RD et al (2007) CCL2 as an important mediator of prostate cancer growth in vivo through the regulation of macrophage infiltration. Neoplasia 9(7):556-562

12. Molloy AP et al (2009) Mesenchymal stem cell secretion of chemokines during differentiation into osteoblasts, and their potential role in mediating interactions with breast cancer cells. Int J Cancer 124(2):326-332

13. Qian BZ et al (2011) CCL2 recruits inflammatory monocytes to facilitate breast-tumour metastasis. Nature 475(7355):222-225

14. Salcedo R et al (2000) Human endothelial cells express CCR2 and respond to MCP-1: direct role of MCP-1 in angiogenesis and tumor progression. Blood 96(1):34-40

15. Saji $\mathrm{H}$ et al (2001) Significant correlation of monocyte chemoattractant protein-1 expression with neovascularization and progression of breast carcinoma. Cancer 92(5):1085-1091

16. Mirolo $M$ et al (2008) Impact of the anti-inflammatory agent bindarit on the chemokinome: selective inhibition of the monocyte chemotactic proteins. Eur Cytokine Netw 19(3):119-122

17. Mora E et al (2012) Bindarit: an anti-inflammatory small molecule that modulates the NFkappaB pathway. Cell Cycle 11(1): 159-169

18. Bhatia $\mathrm{M}$ et al (2008) Treatment with bindarit, an inhibitor of MCP-1 synthesis, protects mice against trinitrobenzene sulfonic acid-induced colitis. Inflamm Res 57(10):464-471

19. Bhatia M et al (2005) Treatment with bindarit, a blocker of MCP1 synthesis, protects mice against acute pancreatitis. Am J Physiol Gastrointest Liver Physiol 288(6):G1259-G1265

20. Ble A et al (2011) Antiproteinuric effect of chemokine C-C motif ligand 2 inhibition in subjects with acute proliferative lupus nephritis. Am J Nephrol 34(4):367-372

21. Guglielmotti A et al (2002) Amelioration of rat adjuvant arthritis by therapeutic treatment with bindarit, an inhibitor of MCP-1 and TNF-alpha production. Inflamm Res 51(5):252-258

22. Ialenti A et al (2011) Inhibition of in-stent stenosis by oral administration of bindarit in porcine coronary arteries. Arterioscler Thromb Vasc Biol 31(11):2448-2454

23. Rulli NE et al (2009) Amelioration of alphavirus-induced arthritis and myositis in a mouse model by treatment with bindarit, an inhibitor of monocyte chemotactic proteins. Arthritis Rheum 60(8):2513-2523

24. Rulli NE et al (2011) Protection from arthritis and myositis in a mouse model of acute chikungunya virus disease by bindarit, an inhibitor of monocyte chemotactic protein-1 synthesis. J Infect Dis 204(7):1026-1030

25. Zhou GX et al (2010) Protective effects of MCP-1 inhibitor on a rat model of severe acute pancreatitis. Hepatobiliary Pancreat Dis Int 9(2):201-207

26. Gazzaniga $S$ et al (2007) Targeting tumor-associated macrophages and inhibition of MCP-1 reduce angiogenesis and tumor growth in a human melanoma xenograft. J Invest Dermatol 127(8):2031-2041

27. D’Angelo A, Garzia L, André A, Carotenuto P, Aglio V, Guardiola O, Arrigoni G, Cossu A, Palmieri G, Aravind L, Zollo M (2004) Prune cAMP phosphodiesterase binds nm23-H1 and promotes cancer metastasis. Cancer Cell 5(2):137-149

28. Roca H, Varsos Z, Pienta KJ (2008) CCL2 protects prostate cancer PC3 cells from autophagic death via phosphatidylinositol 3-kinase/AKT-dependent survivin up-regulation. J Biol Chem 283(36):25057-25073

29. Roca $\mathrm{H}$ et al (2008) CCL2, survivin and autophagy: new links with implications in human cancer. Autophagy 4(7):969-971

30. Yang L et al (2008) Abrogation of TGF beta signaling in mammary carcinomas recruits Gr- $1+\mathrm{CD} 11 \mathrm{~b}+$ myeloid cells that promote metastasis. Cancer Cell 13(1):23-35 
31. Mantovani A (2010) Molecular pathways linking inflammation and cancer. Curr Mol Med 10(4):369-373

32. Balkwill F (2004) Cancer and the chemokine network. Nat Rev Cancer 4(7):540-550

33. Lazennec G, Richmond A (2010) Chemokines and chemokine receptors: new insights into cancer-related inflammation. Trends Mol Med 16(3):133-144

34. Monti P et al (2003) The CC chemokine MCP-1/CCL2 in pancreatic cancer progression: regulation of expression and potential mechanisms of antimalignant activity. Cancer Res 63(21): 7451-7461

35. Fujimoto $\mathrm{H}$ et al (2009) Stromal MCP-1 in mammary tumors induces tumor-associated macrophage infiltration and contributes to tumor progression. Int J Cancer 125(6):1276-1284

36. Ueno $T$ et al (2000) Significance of macrophage chemoattractant protein-1 in macrophage recruitment, angiogenesis, and survival in human breast cancer. Clin Cancer Res 6(8):3282-3289

37. Spano D, Heckc C, De Antonellisa P, Christofori G, Zollo M (2012). Molecular Networks that Regulate Cancer Metastasis. Semin Cancer Biol. doi:10.1016/j.semcancer.2012.03.006. Accessed 30 March 2012

38. Soria G, Ben-Baruch A (2008) The inflammatory chemokines CCL2 and CCL5 in breast cancer. Cancer Lett 267(2):271-285

39. Li X et al (2009) A destructive cascade mediated by CCL2 facilitates prostate cancer growth in bone. Cancer Res 69(4): 1685-1692

40. Lu Y et al (2009) Activation of MCP-1/CCR2 axis promotes prostate cancer growth in bone. Clin Exp Metastasis 26(2): 161-169

41. Soria $\mathrm{G}$ et al (2008) Concomitant expression of the chemokines RANTES and MCP-1 in human breast cancer: a basis for tumorpromoting interactions. Cytokine 44(1):191-200

42. Lu Y et al (2006) Monocyte chemotactic protein-1 (MCP-1) acts as a paracrine and autocrine factor for prostate cancer growth and invasion. Prostate 66(12):1311-1318

43. $\mathrm{Lu} \mathrm{Y}$ et al (2007) CCR2 expression correlates with prostate cancer progression. J Cell Biochem 101(3):676-685

44. Lu Y et al (2007) Monocyte chemotactic protein-1 mediates prostate cancer-induced bone resorption. Cancer Res 67(8):3646-3653

45. Izhak L et al (2012) Dissecting the autocrine and paracrine roles of the CCR2-CCL2 axis in tumor survival and angiogenesis. PLoS One 7(1):e28305

46. van Golen KL et al (2008) CCL2 induces prostate cancer transendothelial cell migration via activation of the small GTPase Rac. J Cell Biochem 104(5):1587-1597

47. Lu Y et al (2007) PTHrP-induced MCP-1 production by human bone marrow endothelial cells and osteoblasts promotes osteoclast differentiation and prostate cancer cell proliferation and invasion in vitro. Int J Cancer 121(4):724-733

48. Qian DZ et al (2010) CCL2 is induced by chemotherapy and protects prostate cancer cells from docetaxel-induced cytotoxicity. Prostate 70(4):433-442

49. Hembruff SL et al (2010) Loss of transforming growth factorbeta signaling in mammary fibroblasts enhances CCL2 secretion to promote mammary tumor progression through macrophagedependent and -independent mechanisms. Neoplasia 12(5): 425-433

50. Loberg RD et al (2007) Targeting CCL2 with systemic delivery of neutralizing antibodies induces prostate cancer tumor regression in vivo. Cancer Res 67(19):9417-9424

51. Zoja C et al (1998) Bindarit retards renal disease and prolongs survival in murine lupus autoimmune disease. Kidney Int 53(3):726-734

52. Guglielmotti A et al (1993) Chronic inflammatory response in the rat can be blocked by bindarit. Biochem Mol Biol Int 29(4): $747-756$
53. Cioli V et al (1992) A new protein antidenaturant agent, bindarit, reduces secondary phase of adjuvant arthritis in rats. $\mathbf{J}$ Rheumatol 19(11):1735-1742

54. Craig MJ, Loberg RD (2006) CCL2 (monocyte chemoattractant protein-1) in cancer bone metastases. Cancer Metastasis Rev 25(4):611-619

55. Rodrigues LR et al (2007) The role of osteopontin in tumor progression and metastasis in breast cancer. Cancer Epidemiol Biomarkers Prev 16(6):1087-1097

56. Rittling SR, Chambers AF (2004) Role of osteopontin in tumour progression. Br J Cancer 90(10):1877-1881

57. Solinas G et al (2010) Tumor-conditioned macrophages secrete migration-stimulating factor: a new marker for M2-polarization, influencing tumor cell motility. J Immunol 185(1):642-652

58. DuPre SA, Redelman D, Hunter KW Jr (2007) The mouse mammary carcinoma 4T1: characterization of the cellular landscape of primary tumours and metastatic tumour foci. Int J Exp Pathol 88(5):351-360

59. Morales JK et al (2010) GM-CSF is one of the main breast tumorderived soluble factors involved in the differentiation of CD11bGr1- bone marrow progenitor cells into myeloid-derived suppressor cells. Breast Cancer Res Treat 123(1):39-49

60. Roland CL et al (2009) Inhibition of vascular endothelial growth factor reduces angiogenesis and modulates immune cell infiltration of orthotopic breast cancer xenografts. Mol Cancer Ther 8(7):1761-1771

61. Melancon MP et al (2010) Targeted imaging of tumor-associated M2 macrophages using a macromolecular contrast agent PG-GdNIR813. Biomaterials 31(25):6567-6573

62. Polfliet MM et al (2006) The rat macrophage scavenger receptor CD163: expression, regulation and role in inflammatory mediator production. Immunobiology 211(6-8):419-425

63. van Dongen M et al (2010) Anti-inflammatory M2 type macrophages characterize metastasized and tyrosine kinase inhibitortreated gastrointestinal stromal tumors. Int J Cancer 127(4): 899-909

64. Sanchez-Martin L et al (2011) The chemokine CXCL12 regulates monocyte-macrophage differentiation and RUNX3 expression. Blood 117(1):88-97

65. Shabo I et al (2008) Breast cancer expression of CD163, a macrophage scavenger receptor, is related to early distant recurrence and reduced patient survival. Int J Cancer 123(4): 780-786

66. Asano $\mathrm{K}$ et al (2011) CD169-positive macrophages dominate antitumor immunity by crosspresenting dead cell-associated antigens. Immunity 34(1):85-95

67. Lau SK, Chu PG, Weiss LM (2004) CD163: a specific marker of macrophages in paraffin-embedded tissue samples. Am J Clin Pathol 122(5):794-801

68. Barral P et al (2010) CD169(+) macrophages present lipid antigens to mediate early activation of iNKT cells in lymph nodes. Nat Immunol 11(4):303-312

69. Allavena P et al (2008) The Yin-Yang of tumor-associated macrophages in neoplastic progression and immune surveillance. Immunol Rev 222:155-161

70. Roy PG, Thompson AM (2006) Cyclin D1 and breast cancer. Breast 15(6):718-727

71. Fu M et al (2004) Minireview: cyclin D1: normal and abnormal functions. Endocrinology 145(12):5439-5447

72. Neumeister P et al (2003) Cyclin D1 governs adhesion and motility of macrophages. Mol Biol Cell 14(5):2005-2015

73. Tsou CL et al (2007) Critical roles for CCR2 and MCP-3 in monocyte mobilization from bone marrow and recruitment to inflammatory sites. J Clin Invest 117(4):902-909 